Portland State University

PDXScholar

Dissertations and Theses

Dissertations and Theses

Fall 1-5-2018

\title{
Strong Coupling Between Photonic Cavities
}

Xiang Wei

Portland State University

Follow this and additional works at: https://pdxscholar.library.pdx.edu/open_access_etds

Part of the Physics Commons

Let us know how access to this document benefits you.

Recommended Citation

Wei, Xiang, "Strong Coupling Between Photonic Cavities" (2018). Dissertations and Theses. Paper 4152. https://doi.org/10.15760/etd.6040

This Thesis is brought to you for free and open access. It has been accepted for inclusion in Dissertations and Theses by an authorized administrator of PDXScholar. Please contact us if we can make this document more accessible: pdxscholar@pdx.edu. 
Strong Coupling Between Photonic Cavities

\section{Xiang Wei}

A thesis submitted in partial fulfillment of the requirements for the degree of

Master of Science

In

Physics

Thesis Committee:

Rolf Koenenkamp, Chair

Andres La Rosa

Pui-Tak Leung

Robert Word

Portland State University

2017 


\section{Abstract}

As the performance of computers has improved dramatically since the 1990s, many interesting photonic crystal properties have been theoretically and experimentally discovered. For example, the strong coupling between photonic crystal cavities was revealed in the 2000s; many groups have successfully fabricated these cavities and verified strong coupling experimentally using silicon. In this thesis, instead of using silicon, we present new results on photonic crystals made by thin indium tin oxide (ITO) layers. Compared to silicon, ITO is not an ideal material to make a photonic crystal because of its comparatively low refractive index and limited transparency. However, it is an interesting model material for experiments in photoemission electron microscopy (PEEM). ITO has a high conductivity that mitigates surface charge-up in an electron microscope and allows electron emission after 2-photon absorption with visible light. We are interested in PEEM because it enables the visualization of the propagation of light with nanometer resolution, i.e., below the optical diffraction limit. In this thesis, we theoretically study ITO photonic crystals in one or two-dimensions with the help of the finite-difference time-domain (FDTD) software. We analyze the electromagnetic field distribution in a manner that the field distributions can directly be compared to experimental PEEM results. We also simulate the strong coupling effect between neighboring cavities and illustrate it in terms of the classical oscillator model. 


\section{Acknowledgements}

This thesis would have been impossible without the help Portland State University Nano Photonic Lab. At here, I would like to thank my advisor Prof. Rolf Koenenkamp for his guiding in theory and practice. I would like to thank Dr. Robert Word and Theodore Stenmark for their advice and introduction of simulation. I also would like to thank every group members for their suggestions. 


\section{Contents}

Abstract $\quad$ i

Acknowledgements $\quad$ ii

List of Figures $\quad$ iv

1 Introduction $\quad \mathbf{1}$

1.1 A brief history of photonic crystals . . . . . . . . . . . . . . . 1

1.2 Simulating electrodynamics using numerical methods . . . . . . . . . 2

1.2.1 The simulation theory . . . . . . . . . . . . 2

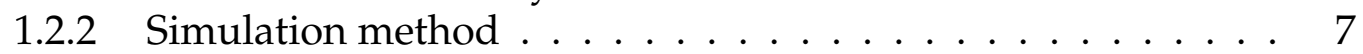

1.3 Types of photonic crystals . . . . . . . . . . . . . . . 9

1.3.1 One-dimensional photonic crystal and the origin of the band

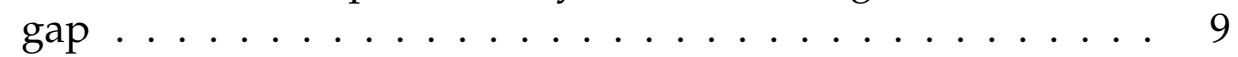

1.3.2 Two-dimensional photonic crystal . . . . . . . . . . . . . . 12

1.3.3 Photonic crystal slabs ................. 18

2 Strong coupling between photonic cavities 22

2.1 Types of photonic cavities . . . . . . . . . . . . . . 22

2.1.1 Point defect photonic cavities . . . . . . . . . . . . 22

2.1.2 Hexagonal defect photonic cavity . . . . . . . . . . . . 23

2.1.3 Line defect photonic cavities . . . . . . . . . . . . . 25

2.2 Coupling between photonic cavities . . . . . . . . . . . 26

2.2.1 The classical model . . . . . . . . . . . . . . . . . . . 27

2.2.2 Coupling strength vs angle and distance . . . . . . . . . . . . 29

2.2.3 ITO based 2D photonic crystal . . . . . . . . . . . . . 32

2.2.4 Strong coupling between L5 photonic cavities . . . . . . . . . 38

2.2.5 Symmetric and antisymmetric modes . . . . . . . . . . . . . . 41

2.2.6 The fringes in the anti-crossing image . . . . . . . . . . 43

3 Conclusion 46

3.1 Future work ....................... 46

$\begin{array}{ll}\text { Bibliography } & 48\end{array}$ 


\section{List of Figures}

1.1 (A) IBM 4 qbits quantum computer chip; (B) UC Berkeley's optical

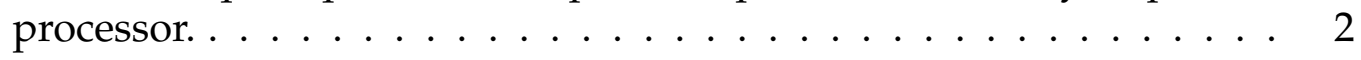

1.2 Leap-frog algorithm. . . . . . . . . . . . . . . . . 4

1.3 Yee Lattice, $H_{x}$ indicates the magnetic field along the $x$ direction, $H_{y}$ indicates the magnetic field along the $y$ direction, $\mathrm{E}$ indicates the electric field point toward the paper. . . . . . . . . . . . 6

1.4 1D photonic crystal. . . . . . . . . . . . . . . . . . 9

1.5 The linear relationship of homogeneous medium. . . . . . . . . . . . 11

1.6 The band structure of coupled oscillators. The yellow bands indicate the band gaps. . . . . . . . . . . . . . . . . . . 12

1.7 A typical 2d square lattice of air holes in photonic crystal. . . . . . . . 13

1.8 (A) TE mode (B) TM modes of a slab photonic crystal. . . . . . . . . . . 14

1.9 (A) TE band structure and (B) TM band structure. . . . . . . . . . 15

1.10 Brillouin zone . . . . . . . . . . . . . . . . . . 15

1.11 (A) Band gap map of ITO; (B) Band gap map of silicon. The green color represents the TE mode and the red color represents the TM mode. . . . . . . . . . . . . . . . 16

1.12 (A) Trianguler lattice; (B) Honeycomb lattice. . . . . . . . . . . . . . . 17

1.13 (A) Structure of ITO slab; (B) 2D photonic crystal TE band structure with light cone overlap. . . . . . . . . . . . . . . . . 19

1.14 (A) Structure of ITO glass; (B) 2D photonic crystal band structure for TE polarization with ITO light cone and glass light cone overlapped. 20

1.15 (A) Structure of ITO glass; (B) TM mode band structure of ITO glass. The red line indicates the frequency of $0.32 \mathrm{c} / \mathrm{a}$. The yellow shadow part indicates leakage. . . . . . . . . . . . . . . 21

2.1 (A) Point defect photonic cavity; (B) High Q point defect photonic cavity. . . . . . . . . . . . . . . . . . 23

2.2 Hexagonal defect photonic cavity. . . . . . . . . . . . . . 24

2.3 Different modes of hexagonal defect photonic cavity. . . . . . . . . . 24

2.4 Line defect photonic cavity. . . . . . . . . . . . . . . . 25

2.5 Different modes of line defect photonic cavity. . . . . . . . . . . 26

2.6 Classical model of coupled oscillators. . . . . . . . . . . . . . . . 27 
2.7 (A) Uncoupled oscillator eigenfrequency plot; (B) Anti-crossing plot of coupled oscillators. The blue line indicates the oscillator A's eigenfrequency, the green line indicates the oscillator B's eigenfrequency. . . . . . . . . . . . . . . .

2.8 (A) Relative cavity positions varied from Angle 1 to Angle 5. The angle $\Theta$ is defined by the direction of upper cavity to the lower cavity VS. the horizontal line. The blue line indicates the direction of leakage indicated by the red line in (B). Various positions of two photonic cavities relative to the photonic lattice as indicated by the angle $\Theta . \ldots \ldots \ldots \ldots$. . . . . . . . . . . . . . . . . . . . . . . 30

2.9 Plot of electric field vs angle. . . . . . . . . . . . . . . . 30

2.10 Relative cavity position varied from distance 1 to distance 5. . . . . . 31

2.11 Plot of electric field vs distance, the unit of distance is a. . . . . . . . . 32

2.12 Spectrum of the L5 photonic cavity with triangular lattice and radius ratio 0.266 . The normalized resonant frequency is determined to be 0.31c/a from the peak. . . . . . . . . . . . . . 33

2.13 (A) Simulation model of L5 photonic cavity; (B) Resonant mode of L5 photonic cavity. . . . . . . . . . . . . . . . . 34

2.14 Two coupled photonic cavity at 30 degrees. . . . . . . . . . 35

2.15 A time variation of two coupled cavities. . . . . . . . . . . . 35

2.16 A plot of upper and lower cavity's energy vs time. . . . . . . . . . . 36

2.17 A simulation model, two cavities at the angle of 30 degrees. . . . . . 38

2.18 Anti-crossing image. . . . . . . . . . . . . . . . 39

2.19 Anti-crossing image with brightness scale overlap. . . . . . . . . . . 40

2.20 Symmetric mode (A); Antisymmetric mode (B). The red color indicates the peak of the electric wave and the blue color indicates the valley of the electric wave. . . . . . . . . . . . . . . . . . . 41

2.21 A frequency scan of the coupling model. . . . . . . . . . . . . . . . 42

2.22 Fringes in one cavity's anticrossing image. . . . . . . . . . . . . . . 43

2.23 Remove one cavity. . . . . . . . . . . . . . . . . . . . . . . . 44

2.24 Move the boundary close to the edge. . . . . . . . . . . 45 


\section{Chapter 1}

\section{Introduction}

\subsection{A brief history of photonic crystals}

Photonic crystals are artificial nanostructures made by higher refractive index materials. The study of the one-dimensional photonic crystal can be traced back to 1880s. In 1887, Lord Rayleigh published a paper titled "The Propagation of Waves through a Medium Endowed with a Periodic Structure" [1] for the first time calculated the photonic crystal band gap in one-dimensions. After him, the study of photonic crystals expands to the two and three-dimensions. In 1987, Yablonovitch and John published two milestone papers and named the system a photonic crystal $[2,3]$. The first photonic crystal in the infrared spectral region was fabricated by Thomas Krauss in 1996 [4]. In 1998, Philip Russell developed the first photonic crystal fiber [5] which realized photonic crystals from theoretical study to application. Today people look for ways to apply light waves in computer processors. Because of the ability to control the flow of light and the ease of fabrication using silicon, photonic crystals became the ideal material to make an optical processor. Figure 1.1A [6] shows a quantum computer chip made by IBM lab using photonic crystals. Figure 1.1B [7] shows an optical computer chip made at UC Berkeley. The chip is made with two processor cores and 850 
photonic components.

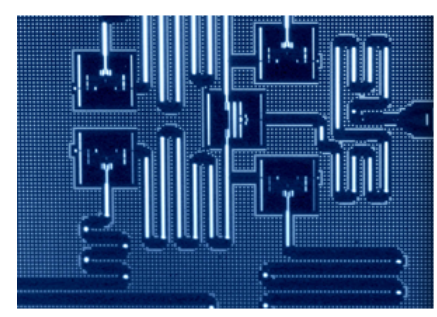

(A)

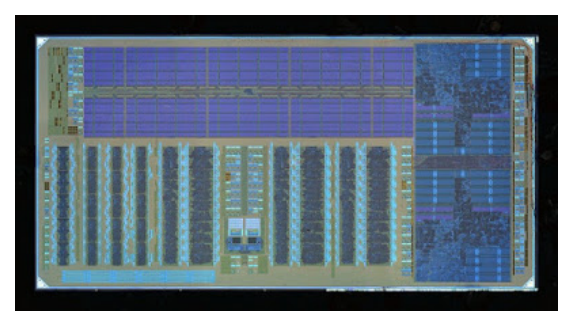

(B)

FIGURE 1.1: (A) IBM 4 qbits quantum computer chip; (B) UC Berkeley's optical processor.

\subsection{Simulating electrodynamics using numerical methods}

\subsubsection{The simulation theory}

The finite-difference time-domain (FDTD) method is a numerical technique used to calculate electromagnetic fields in the time-domain. The Chinese American applied mathematician Kane S. Yee was the first to apply FDTD methods to electromagnetic field calculations in 1966 [8]. After him, the development of FDTD methods in many areas became extremely fast. Almost all FDTD software use the same basic theory from Yee's paper. Nowdays, many FDTD softwares have been developed commercially or as an open source. One of the most popular FDTD software is Meep [9]. The main application of Meep is to calculate the electromagnetic field of photonic crystals. Meep is an open source software developed by MIT. It does not have an UI (User Interface), but it supports several programming languages like Scheme, $\mathrm{C}++$ and Python. Meep also has an MPI (Multiple processing interface) version which supports muti-processing calculations. 
The main idea underlying the FDTD method is to solve Maxwell's equations at every time step and location. The simplest problem is the one-dimensional problem where electromagnetic waves travel in one direction. Suppose we have an electromagnetic wave traveling in a vacuum, Maxwell's equations can be written as:

$$
\begin{gathered}
\nabla \times E=-\frac{1}{c} \frac{\partial H}{\partial t}, \\
\nabla \times H=\frac{1}{c} \frac{\partial E}{\partial t}, \\
\nabla \cdot E=0, \\
\nabla \cdot H=0 .
\end{gathered}
$$

These equations are written in Gaussian units. In the one-dimensional free space problem, we only care about the electromagnetic wave propagating in one direction. For example in the $\mathrm{z}$ direction, the Maxwell equations of becomes:

$$
\begin{gathered}
\frac{\partial E_{x}}{\partial z}=-\frac{1}{c} \frac{\partial H_{y}}{\partial t} \\
\frac{\partial H_{y}}{\partial z}=-\frac{1}{c} \frac{\partial E_{x}}{\partial t} \\
\frac{\partial E_{x}}{\partial z}=0 \\
\frac{\partial H_{y}}{\partial y}=0 .
\end{gathered}
$$

Here we ignore the two Gauss's law equations, since they have no contribution to our calculation. According to Yee's paper, then we can use a central difference 
approximation rewrite the remaining two equations [8]:

$$
\begin{aligned}
\frac{E_{x}^{k+1 / 2}(n+1)-E_{x}^{k+1 / 2}(n)}{\Delta z} & =-\frac{1}{c} \frac{H_{y}^{k+1}(n+1 / 2)-H_{y}^{k}(n+1 / 2)}{\Delta t}, \\
\frac{H_{y}^{k}(n+1 / 2)-H_{y}^{k}(n-1 / 2)}{\Delta z} & =-\frac{1}{c} \frac{E_{x}^{k+1 / 2}(n+1)-E_{x}^{k-1 / 2}(n+1)}{\Delta t} .
\end{aligned}
$$

In these two equations, $E_{x}^{k+1 / 2}(n+1)$ and $H_{y}^{k+1}(n+1 / 2)$ are the electric field and magnetic field in the next time step. We solve these two equations and we get:

$$
\begin{aligned}
& H_{y}^{k+1}(n+1 / 2)=-c \frac{\Delta t}{\Delta z}\left[E_{x}^{k+1 / 2}(n+1)-E_{x}^{k+1 / 2}(n)\right]+H_{y}^{k}(n+1 / 2), \\
& E_{x}^{k+1 / 2}(n+1)=-c \frac{\Delta t}{\Delta z}\left[H_{y}^{k}(n+1 / 2)-H_{y}^{k}(n-1 / 2)\right]+E_{x}^{k-1 / 2}(n+1) .
\end{aligned}
$$

These two equations are the main equations used in the one-dimensional FDTD simulations. First, we use the electric field at $t=0$ to solve for the electric field at time step 1 and then we use the new electric field to solve the magnetic field in time step 1.5. This algorithm is called leap-frog algorithm as shown in Figure 1.2. It is a very common way to solve differential equations using a computer.

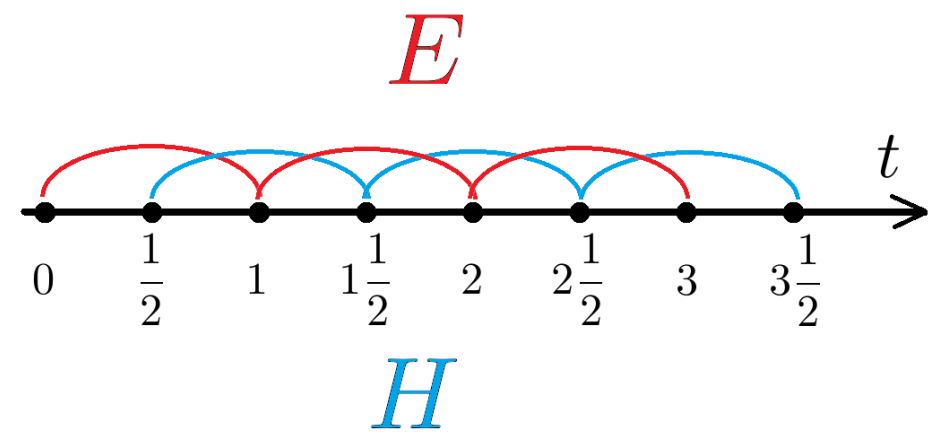

FIGURE 1.2: Leap-frog algorithm. 
If we want to solve a problem in two dimensions, for example, a wave propagating in $x-y$ plane. The electric field is in $z$ direction, the magnetic field is in $\mathrm{x}$ and $\mathrm{y}$ direction. The Maxwell's equations become:

$$
\begin{gathered}
\frac{\partial E_{z}}{\partial y}=-\frac{1}{c} \frac{\partial H_{x}}{\partial t}, \\
\frac{\partial E_{z}}{\partial x}=-\frac{1}{c} \frac{\partial H_{y}}{\partial t}, \\
\frac{1}{c} \frac{\partial E_{z}}{\partial t}=\frac{\partial H_{y}}{\partial x}-\frac{\partial H_{x}}{\partial y} .
\end{gathered}
$$

And similarly, we can apply the central difference method to rewrite these three equations as follows:

$$
\begin{gathered}
\frac{E_{z}^{k+1 / 2}(m+1, n)-E_{z}^{k-1 / 2}(m, n)}{\Delta y}= \\
-\frac{1}{c} \frac{H_{y}^{k+1}(m+1 / 2, n)-H_{y}^{k}(m+1 / 2, n)}{\Delta t}, \\
\frac{E_{z}^{k+1 / 2}(m, n+1)-E_{z}^{k-1 / 2}(m, n)}{\Delta x}= \\
-\frac{1}{c} \frac{H_{y}^{k+1}(m, n+1 / 2)-H_{y}^{k}(m, n+1 / 2)}{\Delta t}, \\
\frac{1}{c} \frac{E_{z}^{k+1 / 2}(m, n)-E_{z}^{k-1 / 2}(m, n)}{\Delta t}= \\
{\left[\frac{H_{y}^{k}(m+1 / 2, n)-H_{y}^{k}(m-1 / 2, n)}{\Delta x}-\frac{H_{x}^{k}(m, n+1 / 2)-H_{x}^{k}(m, n-1 / 2)}{\Delta y}\right] .}
\end{gathered}
$$

These three equations can only be used to calculate the electromagnetic field at one point. To calculate the full electromagnetic field we need to follow a procedure to calculate all the points in the plane. The computation is called the Yee lattice 
method and is illustrated in Figure 1.3:

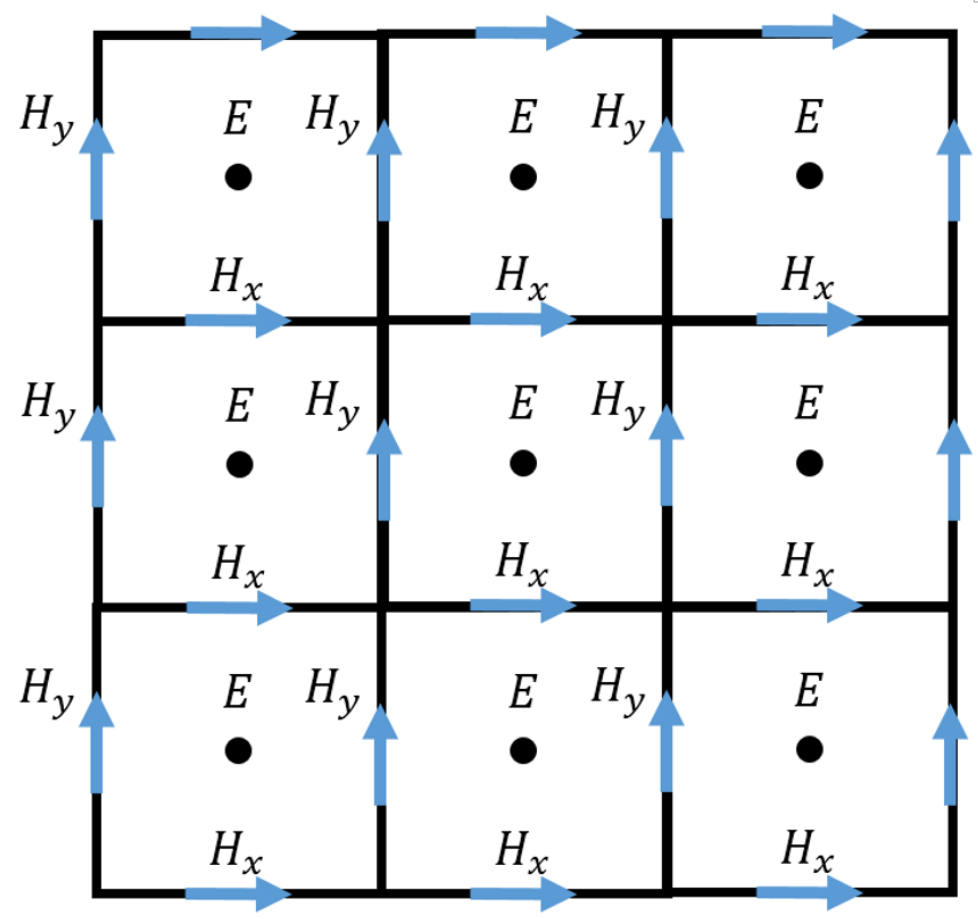

FIGURE 1.3: Yee Lattice, $H_{x}$ indicates the magnetic field along the $\mathrm{x}$ direction, $H_{y}$ indicates the magnetic field along the $y$ direction, E indicates the electric field point toward the paper.

In FDTD method, accuracy depends on the resolution of time and space. The higher the resolution the higher the accuracy. But due to limits of computer speed, memory size, and available time, we cannot use an infinitely high resolution to calcuate the magnetic field. So we need to set a limit for the resolution. A proper resolution can save us a lot of time and also can produce a relative accurate result. Normally, the time resolution should be smaller than $\Delta x / c$, where $\Delta x$ is the space resolution, which should be less than $\lambda / 10$. Here $\lambda$ is the light wavelength inside the material. But in general for two-dimension problems, the most commonly used 
equation to calculate the time step is [10].

$$
\Delta t \leq \frac{\Delta x}{2 c},
$$

for

$$
\Delta x \leq \frac{\lambda}{10} .
$$

In an FDTD simulation the boundary condition is very important. Since we cannot simulate an infinitely large geometry, we always have a boundary. The boundary condition used in our calculation is the absorbing boundary condition. In this kind of boundary, the electromagnetic field will be totally absorbed without any reflection at all frequencies and angles.

\subsubsection{Simulation method}

In our study, we use FDTD software named Meep. Meep is open source software developed by the MIT photonic group [9]. It is software that can only be installed in a UNIX-based machine. It also does not have a user interface (UI), which means that it can only be controlled using a programming language. It supports three programming languages; the two officially supported languages are Scheme and $\mathrm{C}++$. Python can also be supported from a third party website. In our study, we use Scheme, which probably is the easiest way.

Meep can perform 1-D, 2-D, and 3-D simulations. It also supports the Multi-Processing Interface (MPI) standard. Using the MPI version of Meep can boost the speed of simulation several times, depending on the number of 
processors in the computer. In our study, we use a desktop computer with an Ubuntu Linux operating system. Similar to most Linux systems, installing Meep into Ubuntu via the internet can be done just using one single line command (apt-get install meep). It automatically installs the whole Meep related software. To perform the simulation and process the data, we need some other tools to read data file generated by Meep. Meep uses the HDF5 file format to save the simulation results. HDF5 is a data format that can be used to save multi-dimensional 16-bit data. We use MATLAB to analyze the HDF5 file. MATLAB has a function (hd5read) that can be used to read the data set in the HDF5 format. Dependent on the simulation, the data sets read from the HDF5 files can be 1-D matrices, 2-D matrices, and 3-D matrices. In our study, we perform a 2-D simulation, so the result is a 2-D matrix. Depending on the output of the simulation, the data in the matrix can be the amplitude of the electric field or magnetic field. To obtain the proper simulation result, the resolution of the simulation should always be smaller than the $1 / 10$ of the wavelength. Another important tool used in the study is MIT Photonic Bands (MPB). Similar to Meep, it does not have a UI, and it can only be controlled using the Scheme programming language. MPB generates the photonic band structure and photonic band gap map. In our study, we use Python to plot the band structure and band gap map.

In the Meep unit system, the unit of time is $a / c$. Here, $a$ is the periodic constant of the photonic crystal and $c$ is the speed of light. So the unit of frequency in Meep is $c / a$, and this frequency relates to the light frequency. The unit of angular frequency will be $2 \pi c / a$. In our simulation, the space resolution is 
set to $0.1 a$ and the time resolution is set to $0.05 a / c$. The source is a TE point source. The boundary condition we are using is a absorbing boundary condition. The computer is a desktop computer (CPU: AMD 1055T 2.8GHz, 8 GB RAM). The memory required to do the calculation is based on the size and resolution of the simulation model. For our strong coupling model, the lattice is $39 a$ long and $15.6 a$ wide, and the resolution is $0.1 a$. Each point on the simulation model is a $16 \mathrm{bit}$ data. So the total memory required for the $2 \mathrm{D}$ model is about $122 \mathrm{kB}$.

\subsection{Types of photonic crystals}

\subsubsection{One-dimensional photonic crystal and the origin of the band gap}

A one-dimensional photonic crystal is the simplest photonic crystal structure. It is essentially a multilayer film. The periodic structure only varies in one direction as shown in Figure 1.4.

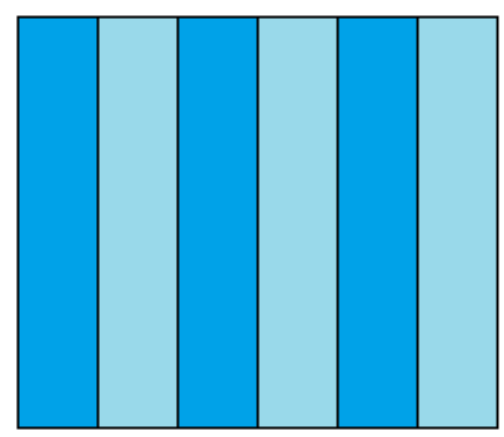

FIGURE 1.4: 1D photonic crystal.

One important property of the photonic crystal is the band gap. To understand the origin of the one-dimensional photonic band gap, we should begin from Maxwell equations [11]. To simplify the problem, we only consider 
linear isotropic, non-magnetic, lossless materials. Maxwell's equations therefore have the following form:

$$
\begin{gathered}
\nabla \cdot H=0, \\
\nabla \cdot \varepsilon E=0, \\
\nabla \times H=\varepsilon_{0} \varepsilon(x) \frac{\partial E}{\partial t}, \\
\nabla \times E=-\mu_{0} \mu \frac{\partial H}{\partial t} .
\end{gathered}
$$

From Maxwell equations one can derive the master equation [11]:

$$
\nabla \times \frac{1}{\varepsilon} \nabla \times H=\left(\frac{\omega}{c}\right)^{2} H .
$$

Then this problem becomes an eigenvalue problem since we are studying an electromagnetic wave in a periodic structure. It must satisfy Bloch's theorem [11]:

$$
H(x, t)=e^{i(k \cdot x-\omega t)} u_{k}(x) .
$$

Where $u_{k}(x)$ is the periodicity of the lattice. In a homogeneous material, the dispersion relationship is a linear:

$$
\omega=\frac{c k}{n} .
$$

where $c$ is the speed of light, $k$ is the wave number, and $n$ is the refractive index. 


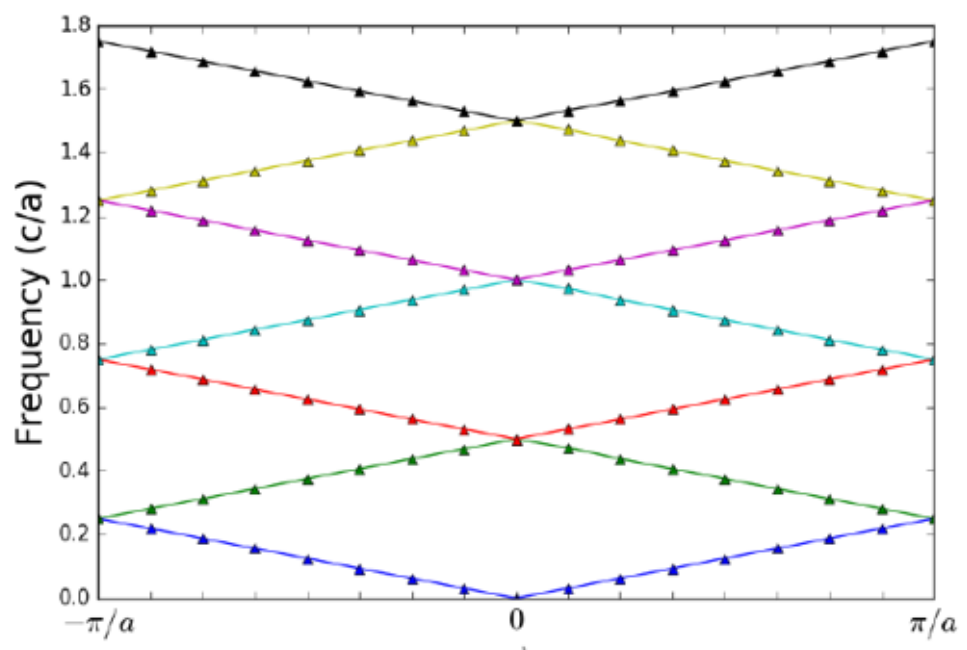

FIGURE 1.5: The linear relationship of homogeneous medium.

Figure 1.5 is the dispersion relationship of homogeneous medium. Here we artificially assign a periodic of $2 \pi / a$. For the $1 \mathrm{D}$ photonic crystal, one can solve the master equation and find the dispersion relationship. Different from the homogeneous material, the dispersion relation is shown in Figure 1.6: 


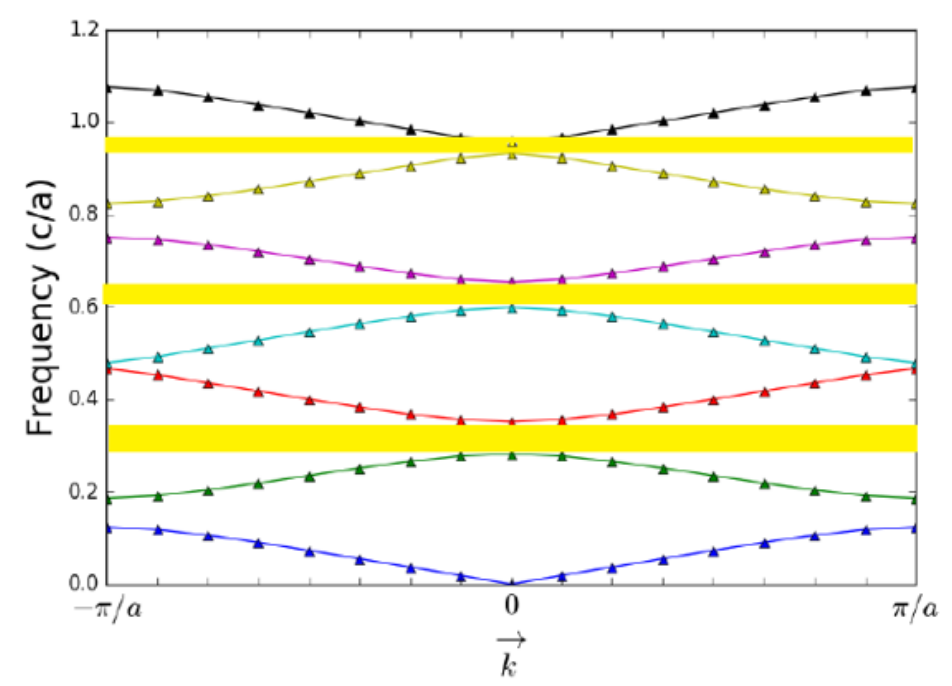

FIGURE 1.6: The band structure of coupled oscillators. The yellow bands indicate the band gaps.

If we artificially fold the dispersion relationship at $\pi / a$, we can find a band gap at $k=\pi / a$ and $k=-\pi / a$. There are many applications of one-dimensional photonic crystals. People have already used them to increase the brightness of LEDs, make optical filters, and make laser oscillator chambers [12, 4, 13]

\subsubsection{Two-dimensional photonic crystal}

A two-dimensional photonic crystal has a periodic dielectric constant in the $\mathrm{x}$ and $\mathrm{y}$ directions, but not in the $\mathrm{z}$ direction. In the $\mathrm{z}$ direction, the dielectric constant is constant. Similar to the one-dimensional photonic crystal, a two-dimensional photonic crystal can prevent propagation in the periodically structured directions, i.e. the $\mathrm{x}$ and $\mathrm{y}$ direction. A typical example of a two-dimensional photonic crystal is a square lattice of air holes as shown in Figure 1.7. The square lattice air hole photonic crystal is infinite in the $\mathrm{z}$ direction. For a different diameter of the air 
holes and a different dielectric constant, the photonic crystal will have a different band gap.

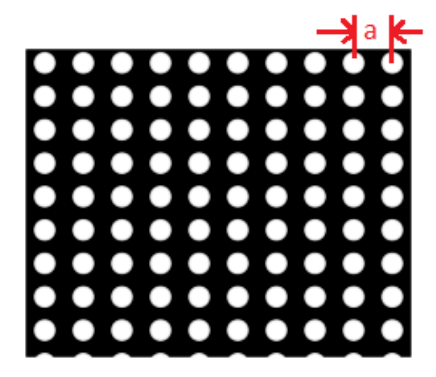

FIGURE 1.7: A typical 2d square lattice of air holes in photonic crystal.

For electromagnetic waves in a $2 \mathrm{D}$ photonic crystal, we need to treat Transverse Electric (TE) and Transverse Magnetic (TM) modes separately. For TE modes, the electric field is inside the 2D photonic crystal plane. But for a TM wave, the magnetic field is inside the $2 \mathrm{D}$ photonic crystal plane, as shown in Figure 1.8 


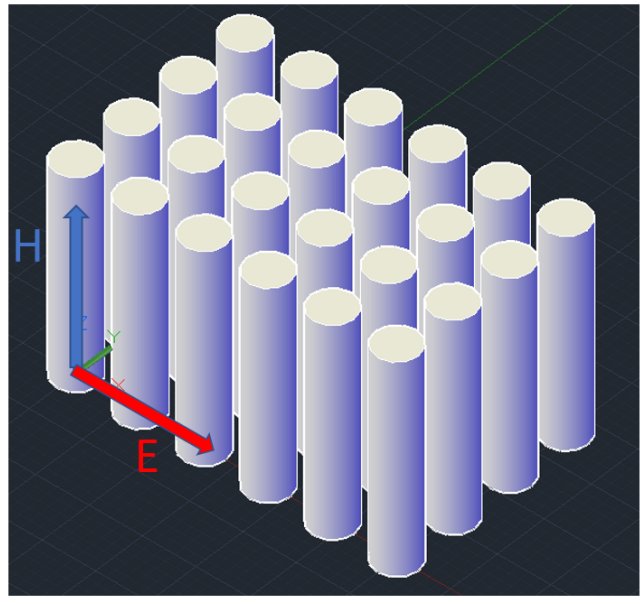

(A)

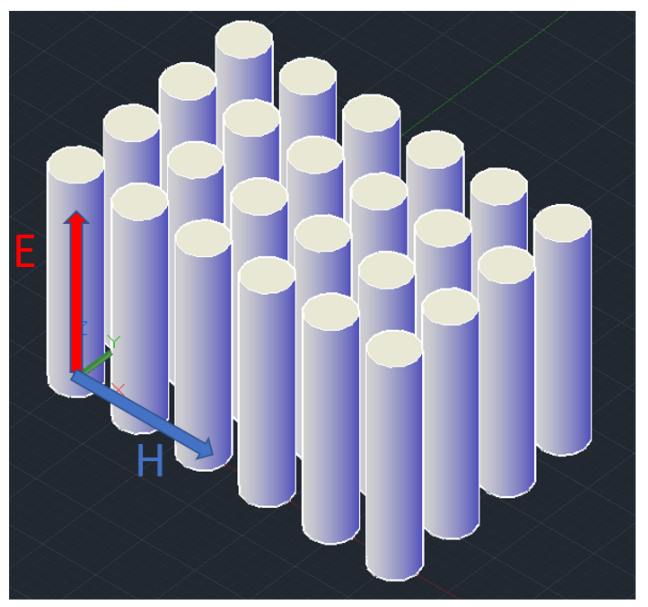

(B)

FIGURE 1.8: (A) TE mode (B) TM modes of a slab photonic crystal.

Because the electric field and magnetic field have different directions between TE and TM waves, the TE and the TM modes will have different propagation properties. Therefore, the photonic band structure for TE and TM modes will also be different. Figure 1.9 is the band structure of a triangular lattice photonic crystal. The radius of each air hole is $0.266 a$ and the refractive index of the material is 2.18. Each band in the Figure 1.9 represent the mode that can propogate through the photonic crystal. The region between two bands in which no mode can propogate through the photonic crystal is the band gap. For the same 2D photonic crystal, the TE mode band structure and TM mode band structure is different. If we overlap the TE and the TM mode band structure, and we can still find a band gap, the photonic crystal is said to have a complete band gap. Complete bandgap refers to a bandgap in all directions and all polarizations. Inside a complete band gap, no matter what kind of polarization the 
electromagnetic wave has, it cannot propagate through the photonic crystal.

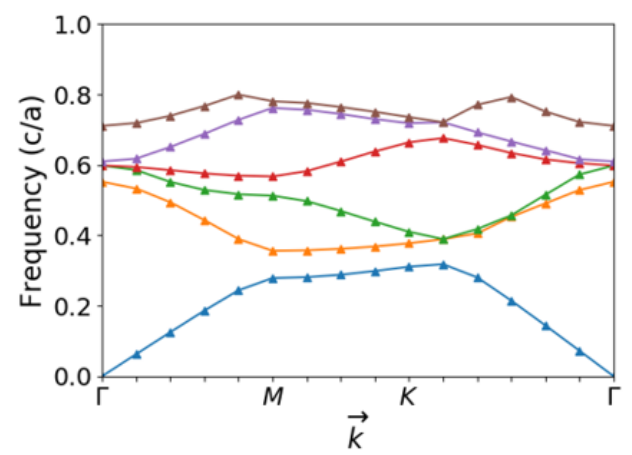

(A)

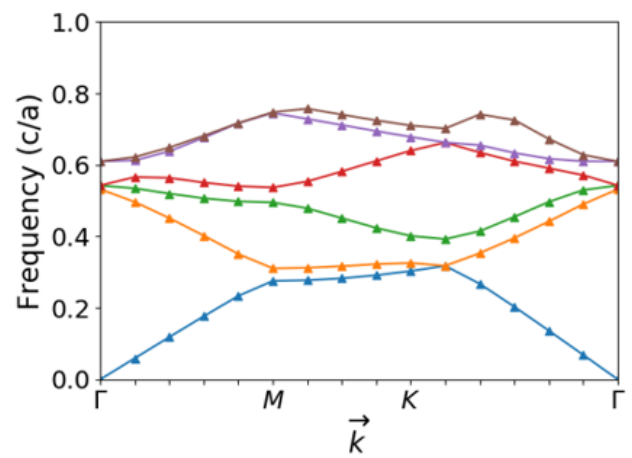

(B)

FIGURE 1.9: (A) TE band structure and (B) TM band structure.

In Figure 1.9A and Figure 1.9B the vertical axis is the frequency in unit of $c / a$, and the horizontal axis is the wavenumber $\mathrm{k}$ in photonic crystal given by $k=$ $2 \pi n / \lambda$ with $n$ the refractive index of photonic crystal. All these plots are plotted using the data generated by MPB. In Figure 1.9, $\mathrm{k}$ is plotted for certain directions of the Brillouin zone. For example, the $\Gamma$ point is at the center of the Brillouin zone, while the $M$ point is on the edge of Brillouin zone, and the $K$ point is in the corner of the Brillouin zone, as shown in Figure 1.10.

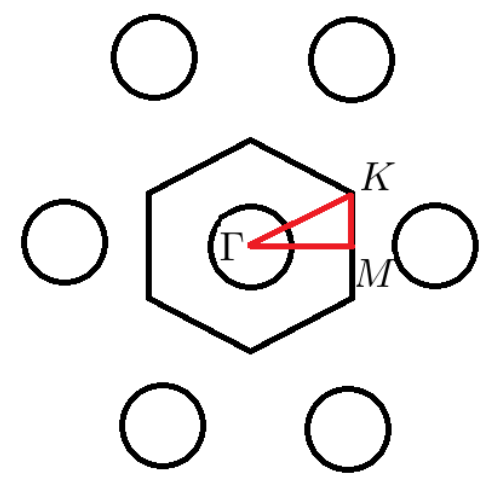

FIGURE 1.10: Brillouin zone 
Hence, the first section in the plots describes the behavior of wave vector $k$ along the direction from $\Gamma$ to $M$. The second section describes the behavior of $k$ along the direction from $M$ to $K$. Then the last section describes from $K$ back to $\Gamma$. Figure 1.9a is the TE mode band structure. We find a band gap with a center frequency of $0.32 c / a$. For the TM band structure we cannot find a band gap in Figure $1.9 \mathrm{~b}$. We can also vary the radius ratio and find a range of complete band gaps. Comparing band gap range of the TE mode to TM mode, we find that the TM band gaps are narrower.

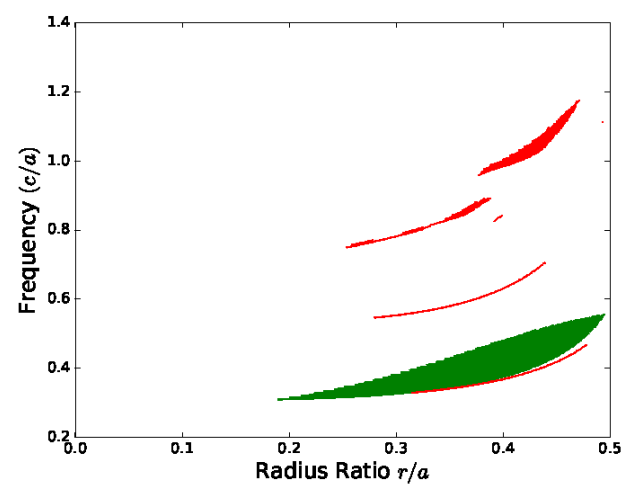

(A)

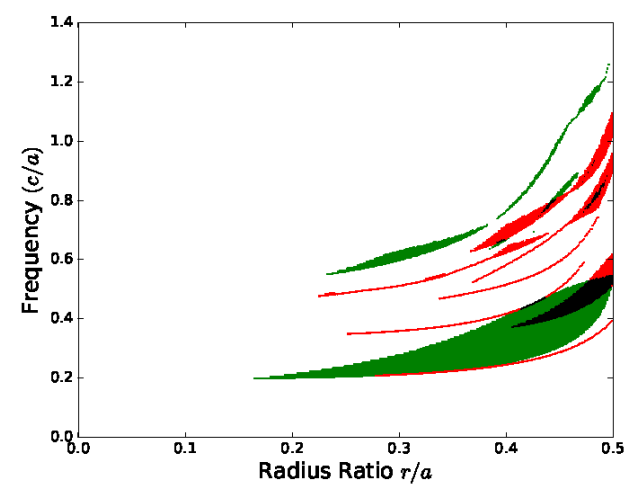

(B)

FIGURE 1.11: (A) Band gap map of ITO; (B) Band gap map of silicon. The green color represents the TE mode and the red color represents the TM mode.

Based on the frequency and the laser wavelength, we can determine a periodic constant

$$
f=0.32 \frac{c}{a} .
$$

in which

$$
a=0.32 \frac{c}{f}=0.32 \lambda .
$$


In a future experiment, we plan to use a laser with $\lambda=400 \mathrm{~nm}$,

$$
a=0.32 \times 400 \mathrm{~nm}=128 \mathrm{~nm} .
$$

Thus, for a wavelength of $400 \mathrm{~nm}$ we need a lattice periodic constant of $128 \mathrm{~nm}$ for the triangular photonic crystal lattice. In order to put the laser energy in the band gap region, according to the simulation result, we need to set the radius ratio equal to $34 \mathrm{~nm}$ :

$$
r=0.266 \times 128 \mathrm{~nm}=34 \mathrm{~nm} .
$$

Using these parameters, we can use our simulation to guide our future experimental work.

The lattice type of the photonic crystal is also an important factor for the photonic crystal band gap. The most commonly used two-dimensional photonic crystal lattice is the triangular lattice as shown in Figure 1.12A. Another widely used photonic crystal lattice is the honeycomb lattice (Figure 1.12B).

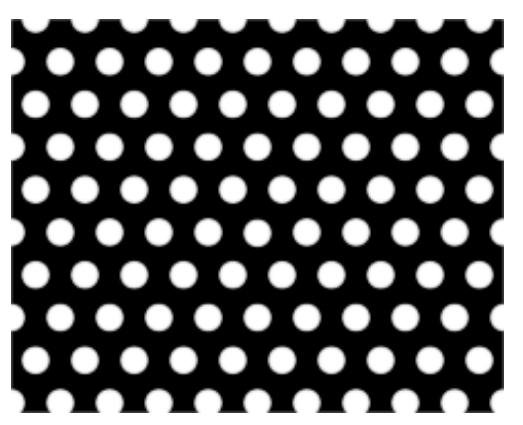

(A)

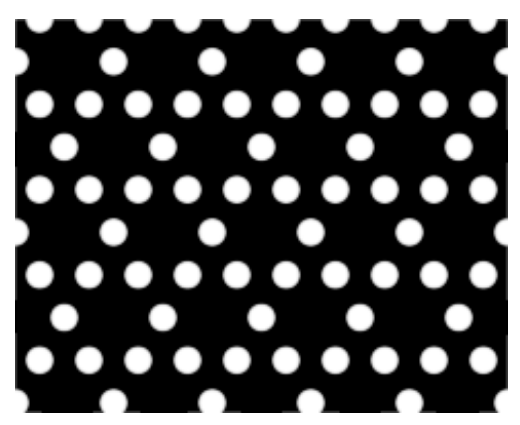

(B)

FIGURE 1.12: (A) Trianguler lattice; (B) Honeycomb lattice. 


\subsubsection{Photonic crystal slabs}

A two-dimensional photonic crystal is an ideal photonic crystal structure to study properties of photonic crystals. But in the real world, we cannot build a two-dimensional structure. Photonic crystal slabs of finite thickness are the best approximation of two-dimensional photonic crystal in the real world. Similar to two-dimensional photonic crystals, photonic crystal slabs have a periodic structure in two directions, but in the third direction there is no periodic structure. Since it is a three-dimensional structure, we cannot directly use calculation results we obtained from the two-dimensional photonic crystal. Our experimental material is Indium Tin Oxide (ITO) film, which is on glass. This is an asymmetric system. For simplicity, we assume an ITO layer surrounded by air as shown in Figure 1.13a. According to Snell's law, when the tangential component (the component parallel to the surface) of the k-vector is smaller than $2 \pi / \lambda$, the light can escape from the slab. When the tangential component of k-vector is larger than $2 \pi / \lambda$, the light cannot escape from the slab [11]. Our calculation is based on two dimensional model, but it is not sensitive to the top and bottom materials. We can plot the light line and overlap it with our band structure as shown in Figure $1.13 \mathrm{~b}$. With inclusion of the light cone, the photonic crystal no longer have a complete band gap in the TE mode, but it still can have a band gap in a limited range of directions. The model we use to plot Figure $1.13 \mathrm{~b}$ is a triangular lattice photonic crystal as shown in Figure 1.12 with $r=0.266 \mathrm{a}$ and refractive index equal to 2.18 . 


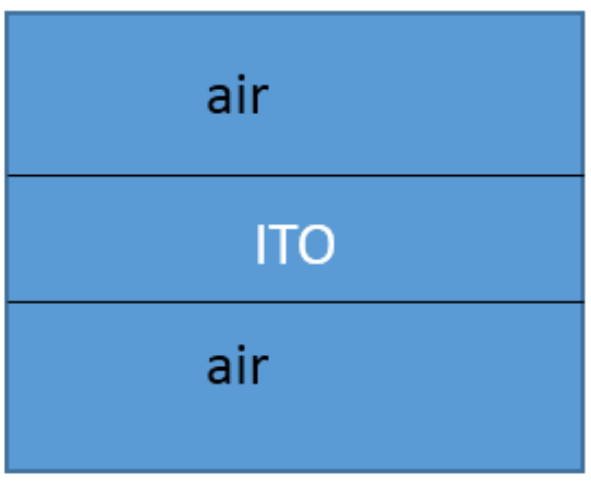

(A)

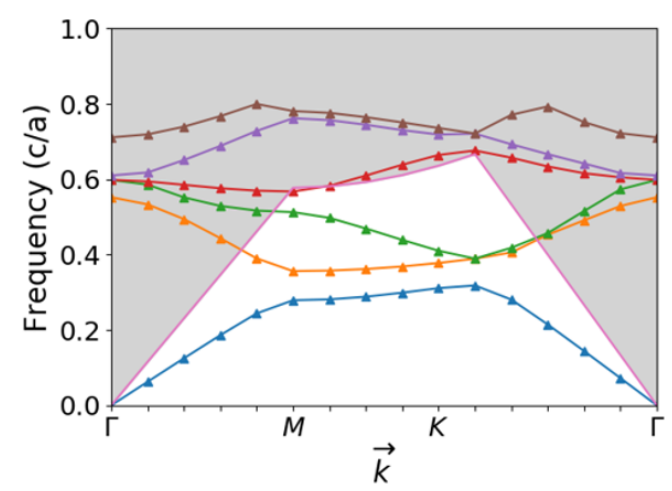

(B)

FIGURE 1.13: (A) Structure of ITO slab; (B) 2D photonic crystal TE band structure with light cone overlap.

However, the structure we are going to use in the future experiments is more complex. We have an asymmetric structure. So on the top of ITO will be air, and at the bottom of the ITO will be glass as shown in Figure 1.14a. Different from the symmetric model we discussed, the asymmetric model has to overlap two light cones, one from the ITO and another one from the glass. As shown in Figure 1.14b, the top light line comes from ITO, and the bottom light line comes from the glass. So the localized mode can only exist between these two lines [14], the white region in Figure 1.14b. Figure 1.14b is using the same lattice as Figure 1.13b. 


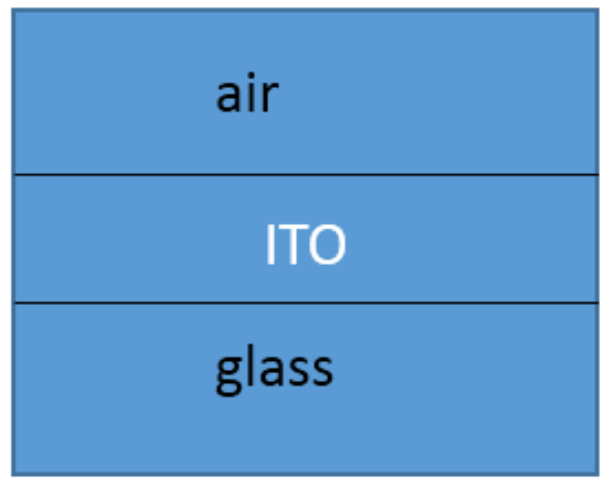

(A)

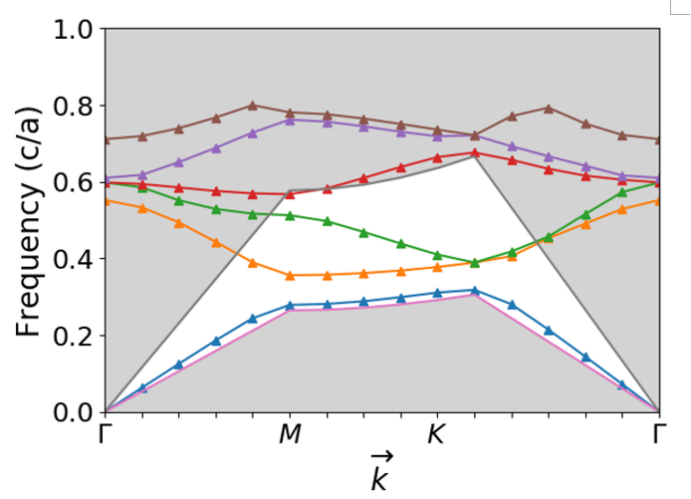

(B)

FIGURE 1.14: (A) Structure of ITO glass; (B) 2D photonic crystal band structure for TE polarization with ITO light cone and glass light cone overlapped.

People have already explored the strong coupling effect of the TE mode[15]. However, the photonic cavity may not need a complete bandgap to form a resonant mode. Like an optical cavity made with mirrors, the strongest reflection only need exist in one direction. In the TM mode band structure, leakage occurs in somewhere between $K$ and $\Gamma$ direction. The leakage is indicated by the yellow shadow part in Figure 1.15. We may therefore investigate the properties of an ITO photonic cavity for the TM mode instead of the TE mode. 


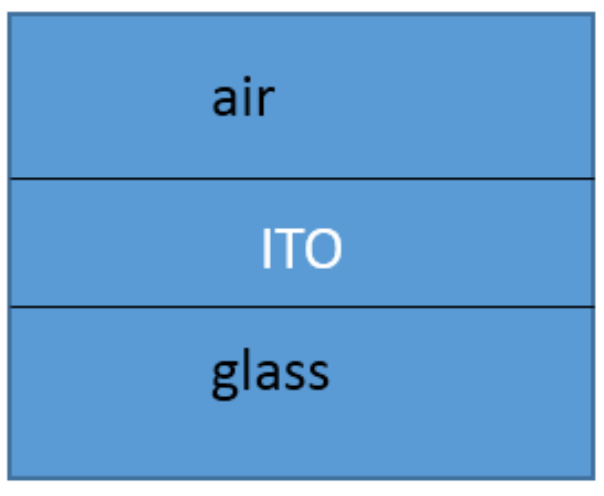

(A)

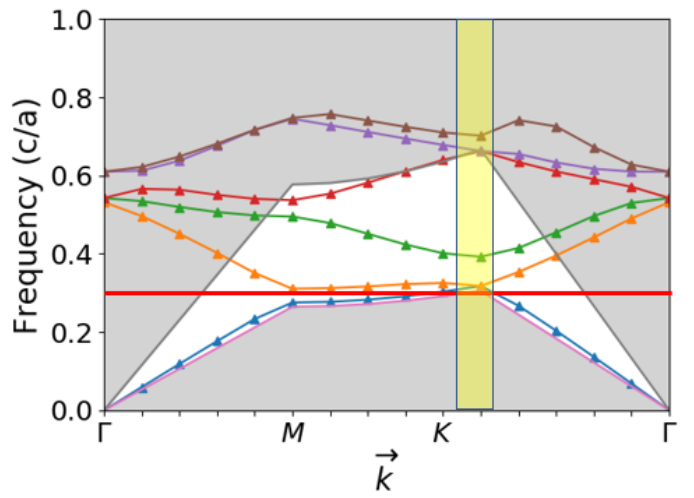

(B)

FIGURE 1.15: (A) Structure of ITO glass; (B) TM mode band structure of ITO glass. The red line indicates the frequency of $0.32 \mathrm{c} / \mathrm{a}$. The yellow shadow part indicates leakage. 


\section{Chapter 2}

\section{Strong coupling between photonic cavities}

\subsection{Types of photonic cavities}

An optical cavity, resonating cavity, or optical resonator is an arrangement of mirrors that forms a standing wave cavity resonator for light waves. Because of the photonic crystal band gap we can arrange the structure of the photonic crystal to form an optical cavity. Similar to a traditional optical cavity, light confined in a photonic crystal cavity forms standing waves for certain resonance wavelengths. The spatial patterns generated by the standing waves are called resonant modes.

\subsubsection{Point defect photonic cavities}

A point defect photonic cavity is the simplest kind of photonic crystal cavity. It can be created by removing a single hole in a photonic crystal slab [11]. Figure 2.1A shows a point defect photonic cavity in an air hole photonic crystal structure. 


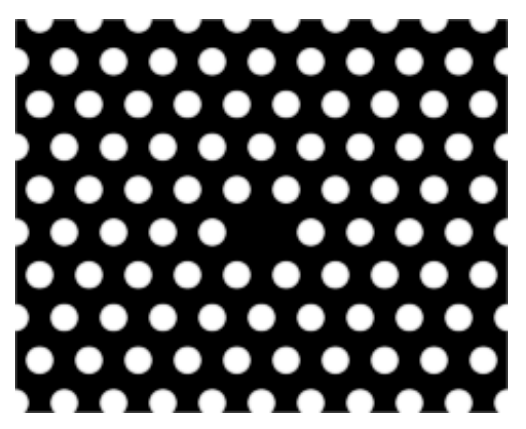

(A)

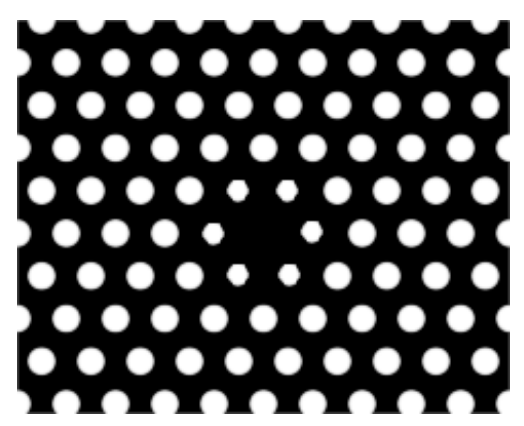

(B)

FIGURE 2.1: (A) Point defect photonic cavity; (B) High Q point defect photonic cavity.

Because we put a point defect in a photonic crystal, the band gap of the photonic crystal can prohibit electromagnetic waves propagate through the photonic crystal structure. But once we put a point defect inside the photonic crystal the translational symmetry of the photonic crystal slab will be broken. This will allow localized modes to appear inside the photonic crystal band gap. Since the remnant part of the photonic crystal slab still maintains translational symmetry, light is still forbidden in the remnant part of the photonic crystal slab. This creates an optical cavity. Because of the small dimensions of the point defect photonic cavity, the mode volumes are very small. Note that a common way to increase the quality factor of the point defect photonic cavity is to change the hole size around the photonic crystal [16], as shown in Figure 2.1B. The radius of the air holes is $0.266 a$ and the radius of the air holes around the cavity is $0.15 a$.

\subsubsection{Hexagonal defect photonic cavity}

Similar to a point defect photonic crystal cavity, a hexagonal defect photonic cavity can be treated as a large size point defect photonic crystal cavity [11]. An example 
of hexagonal defect photonic cavity can be found in Figure 2.2

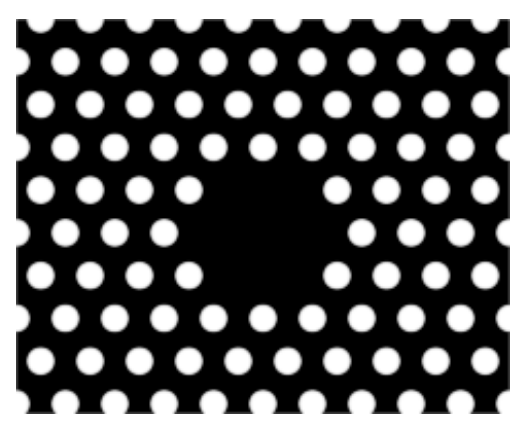

FIGURE 2.2: Hexagonal defect photonic cavity.

The modes in a hexagonal defect photonic cavity are complex due to different air hole radius. We use Meep to run an FDTD simulation on a hexagonal defect photonic crystal and find some interesting modes as shown in Figure 2.3.

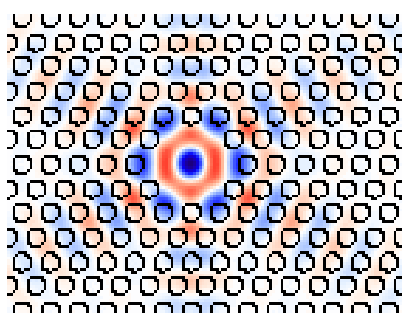

(A)

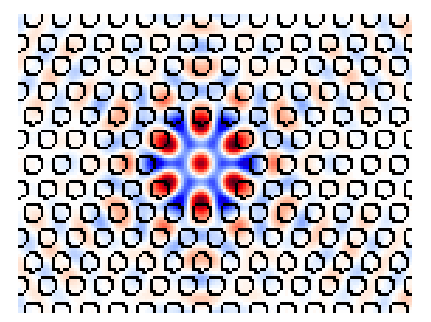

(B)

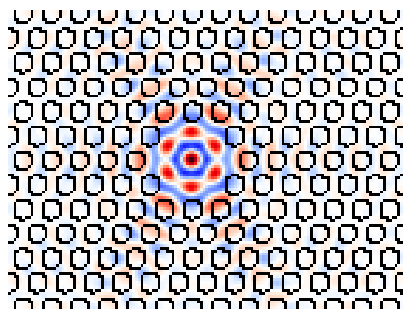

(C)

FIGURE 2.3: Different modes of hexagonal defect photonic cavity.

All the models are using a triangular lattice with refractive index equal to 2.18. The source is in the center of the cavity with TE polarization. These modes are not localized modes. The center frequency is not in the badgap. Therefore energy leaks in some directions. For the case shown in Figure 2.3A, the hole radius is $0.288 a$ and the wavelength is $3.25 a$. For the case shown in Figure $2.3 \mathrm{~B}$, the hole radius is $0.288 a$ 
and the wavelength is $2.59 a$. For the case shown in Figure $2.3 \mathrm{C}$, the hole radius is $0.304 a$ and the wavelength is $1.89 a$.

\subsubsection{Line defect photonic cavities}

A line defect photonic cavity can be treated as several points defect photonic cavity combined [11]. It is very similar to a waveguide closed at both ends. An example of a line defect photonic cavity can be found in Figure 2.4

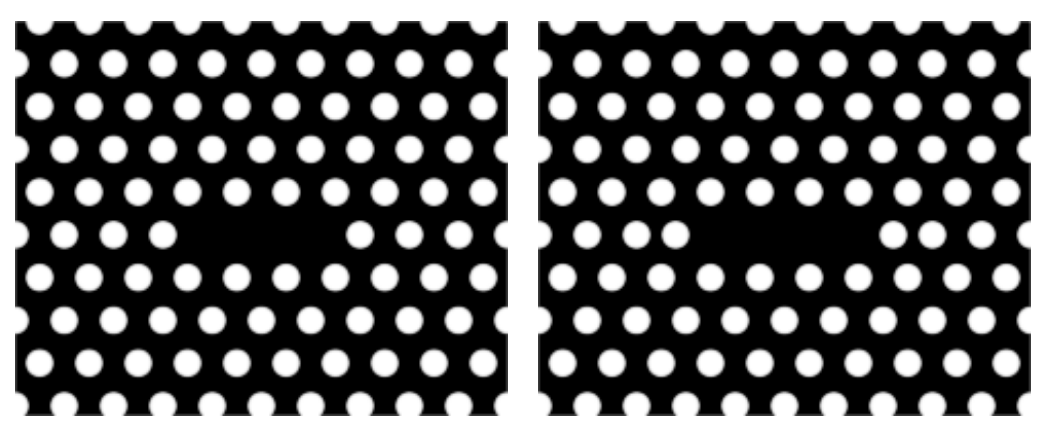

(A)

(B)

FIGURE 2.4: Line defect photonic cavity.

The simplest way to increase the $Q$ factor of a line defect photonic cavity is to slightly move the position of the two air holes at each end of the photonic cavity as shown in Figure 2.4 [17]. A similar property can be found in a plane-parallel optical cavity. The holes at each end of the photonic cavity are moved out $0.196 a$. We ran an FDTD simulation on an L5 cavity (a line defect photonic cavity with five missing air holes). At different frequencies, the cavity has different modes as shown in Figure 2.5: 


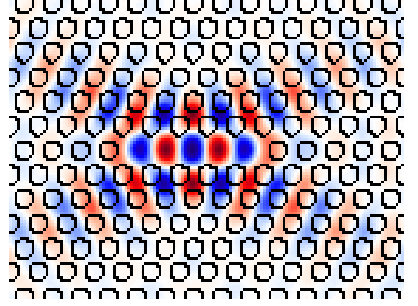

(A)

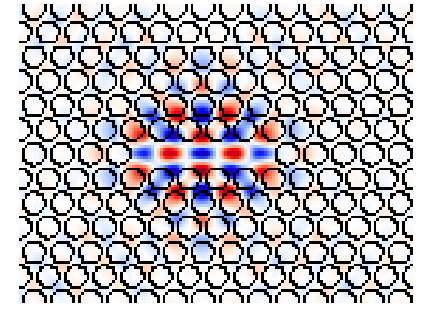

(B)

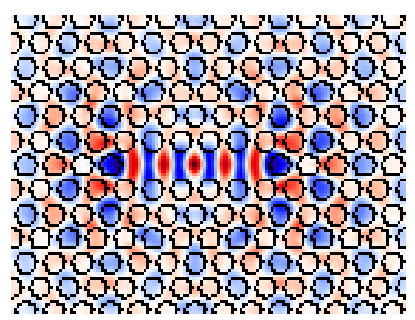

(C)

FIGURE 2.5: Different modes of line defect photonic cavity.

All the models are using a triangular lattice with refractive index equal to 2.18. The source is in the center of the cavity with TE polarization. These modes are also not localized modes, the center frequency is not in the badgap, so there are also energy leakages in some directions. For the case shown in Figure 2.5A, the hole radius is $0.3 a$ and the wavelength is $3.25 a$. For the case shown in Figure 2.5B, the hole radius is $0.324 a$ and the wavelength is $1.84 a$. For the case shown in Figure $2.5 \mathrm{C}$, the hole radius is $0.328 a$ and the wavelength is $2.28 a$.

\subsection{Coupling between photonic cavities}

The energy transfer between two quantum systems is typically studied in weak coupling and strong coupling regimes. In the weak coupling case, the energy transfer rate from each of the cavities to the environment is larger than the transfer rate between the two cavities. But for the strong coupling case, the energy transfer rate between the two cavities are equal and the coupling rate to the environment is small compare to the rate between the two cavities. A notable characteristic feature of strong coupling is energy splitting, which can be understood using a coupled oscillator. 


\subsubsection{The classical model}

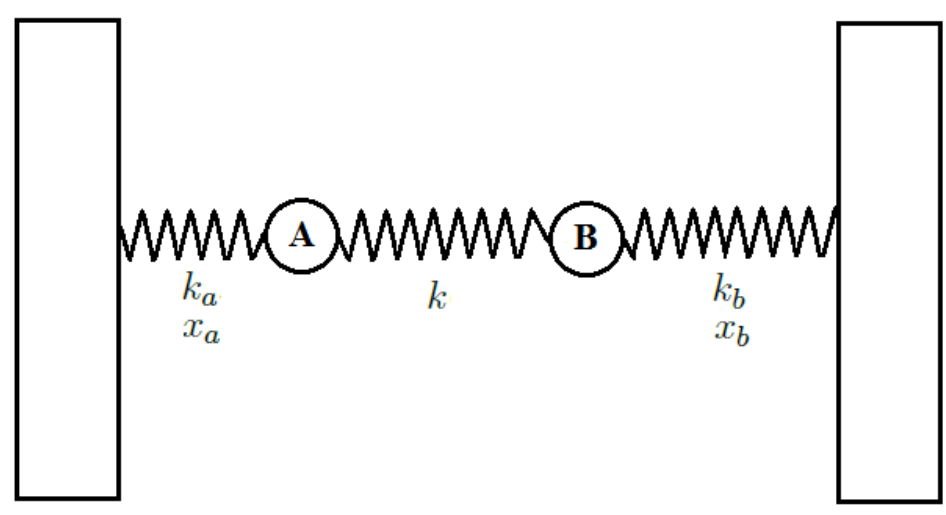

FIGURE 2.6: Classical model of coupled oscillators.

From Lukas Novotny's paper [18], the strong coupling effect can be studied using a classical model. In Figure 2.6, there is a two oscillator system, which is similar to the two cavity system. The mass of Oscillator $\mathrm{A}$ is $M_{a}$, and its spring constant is $k_{a}$. The mass of Oscillator $\mathrm{B}$ is $M_{b}$, and its spring constant is $k_{b}$. The coupling spring constant is $k$. The equations of motion of this system are:

$$
\begin{aligned}
& k_{a} x_{a}+m_{a} \ddot{x}_{a}+k\left(x_{a}-x_{b}\right)=0, \\
& k_{b} x_{b}+m_{b} \ddot{x}_{b}-k\left(x_{a}-x_{b}\right)=0 .
\end{aligned}
$$

Here, $x_{a}$ and $x_{b}$ are displacement of two oscillators. From Lukas Novotny's paper, we could solve the equations and obtain two different eigenfrequencies:

$$
\omega_{a}^{2}=\frac{1}{2}\left[\omega_{a}^{2}+\omega_{b}^{2}+\sqrt{\left(\omega_{a}^{2}-\omega_{b}^{2}\right)^{2}+4 \Gamma^{2} \omega_{a} \omega_{b}}\right]
$$




$$
\omega_{b}^{2}=\frac{1}{2}\left[\omega_{a}^{2}+\omega_{b}^{2}-\sqrt{\left(\omega_{a}^{2}-\omega_{b}^{2}\right)^{2}+4 \Gamma^{2} \omega_{a} \omega_{b}}\right] .
$$

where $\Gamma$ is:

$$
\Gamma=\frac{\sqrt{k / m_{a}} \sqrt{k / m_{b}}}{\sqrt{\omega_{a 0} \omega_{b 0}}}
$$

and

$$
\begin{aligned}
& \omega_{a}=\sqrt{\left(k_{a}+k\right) / m_{a}}, \\
& \omega_{b}=\sqrt{\left(k_{b}+k\right) / m_{b}} .
\end{aligned}
$$

In this calculation, we ignored damping in the analysis. We now tune one of these oscillators' spring constant $k_{b}$. We keep oscillator A's spring constant $k_{a}$ constant (so $k_{a}=k_{0}$ ). We keep A\&B's mass equal to $m_{0}$, and we express oscillator B's spring constant as $k_{b}=k_{0}+\Delta k$. We then change $\Delta k$ from $-k_{0}$ to $k_{0}$. We can then plot the uncoupled relation and use Equation 2.3 and 2.4 to plot the coupled relationship, as shown in Figure 2.7.

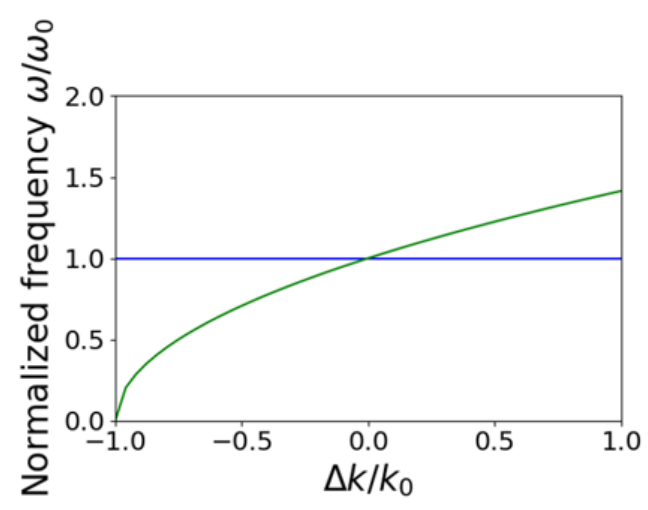

(A)

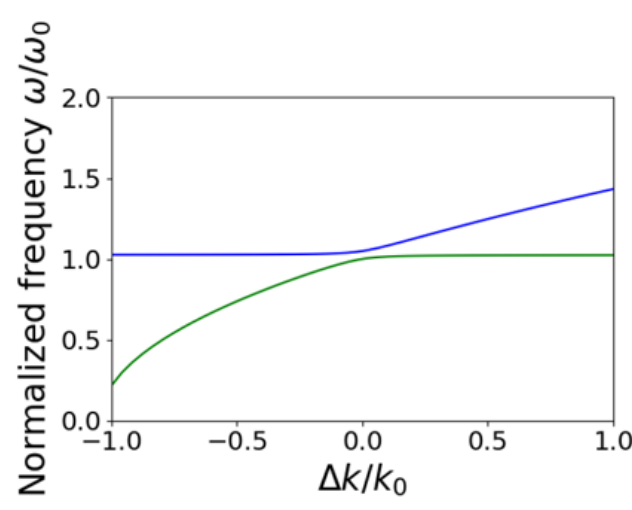

(B)

FIGURE 2.7: (A) Uncoupled oscillator eigenfrequency plot; (B) Anti-crossing plot of coupled oscillators. The blue line indicates the oscillator A's eigenfrequency, the green line indicates the oscillator B's eigenfrequency. 
In Figure 2.7, horizontal axis is the ratio of $\Delta k$ and $k_{0}$, and the vertical axis is the normalized eigenfrequencies. The frequency is normalized to $\omega / \omega_{0}$, Where $\omega_{0}=\sqrt{k_{0} / m_{0}}$. For the uncoupled oscillator in Figure 2.7A, the eigenfrequencies of the two oscillators cross each other at the center of the plot. For the coupled oscillator in Figure 2.7B, the eigenfrequencies avoid each other at the center of the plot. Figure 2.7B shows an anti-crossing behavior.

\subsubsection{Coupling strength vs angle and distance}

In a system of two optical cavities, the angle between two photonic cavities is very important. This is because the energy of a cavity leaks outward in preferred directions. In order to find the strongest coupling angle for an experiment, we run several FDTD simulations and change the angle between the two cavities, as shown in Figure 2.8A. We calculated the average absolute electric field amplitude in the left cavity over several cycles and varied the angle $\Theta$ as shown in Figure 2.8A. 


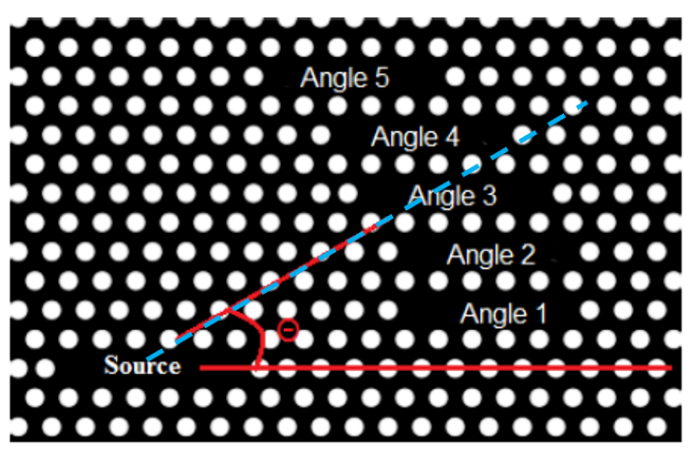

(A)

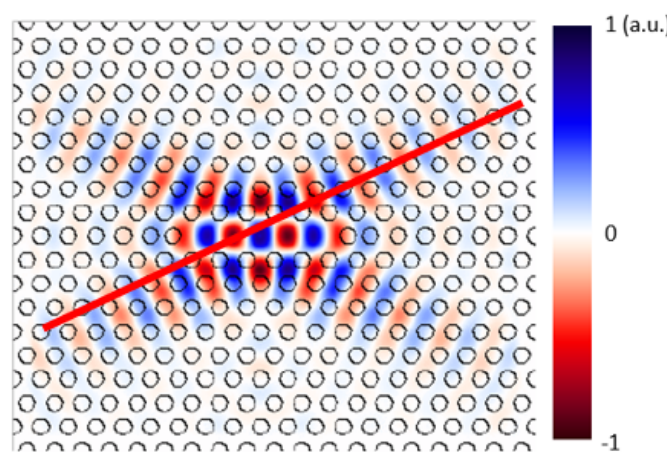

(B)

FIGURE 2.8: (A) Relative cavity positions varied from Angle 1 to Angle 5. The angle $\Theta$ is defined by the direction of upper cavity to the lower cavity VS. the horizontal line. The blue line indicates the direction of leakage indicated by the red line in (B). Various positions of two photonic cavities relative to the photonic lattice as indicated by the angle $\Theta$.

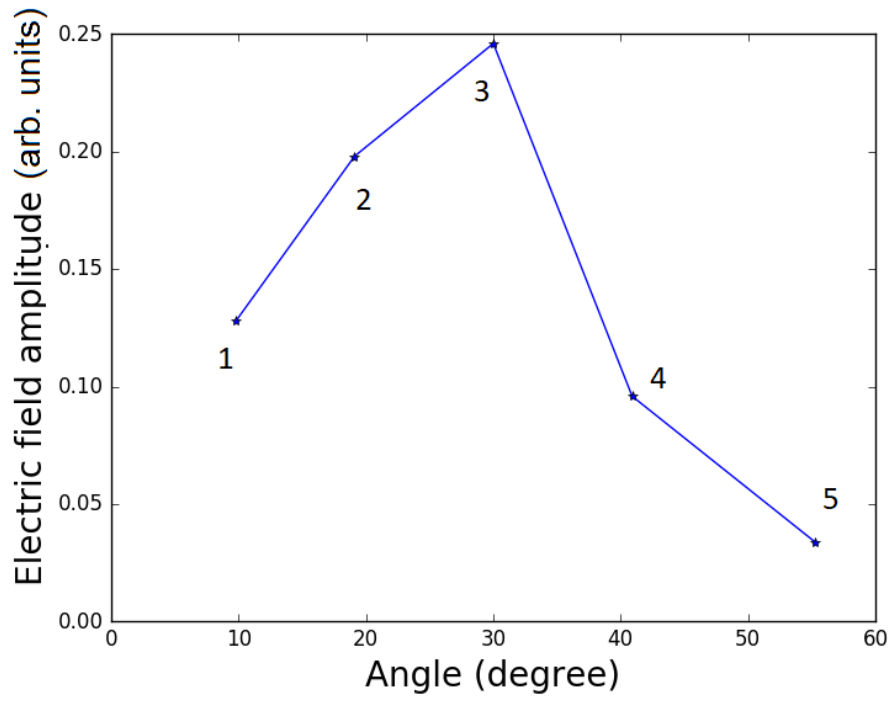

FIGURE 2.9: Plot of electric field vs angle.

We can see as the angle $\Theta$ changes, the electric field amplitude inside the upper cavity also changes. Angle 3 leads to the maximum amplitude of electric 
field, which corresponding to the highest coupling strength. From Figure 1.15 we cannot find a band gap around the $K$ point. The TM mode electromagnetic wave leaks in this direction $(\Gamma-K)$. The $\Gamma-K$ direction of triangular lattice photonic crystal (shown in Figure 1.12) correspond to the $\Theta=30$ degrees as shown in Figure 2.8A.

Another important variable between these two photonic cavities is their separation distance. The distance between two cavities cannot be too far. This results a weaker coupling strength. Also, the distance cannot be too short. A short distance will merges the two photonic cavities into one photonic cavity. In order to verify the relationship between the coupling strength and the distance, we ran several simulations as shown in Figure 2.10. The distances labeled 1-5 represent five different simulations. We calculated the average energy density in the upper cavity. The result is shown in Figure 2.11.

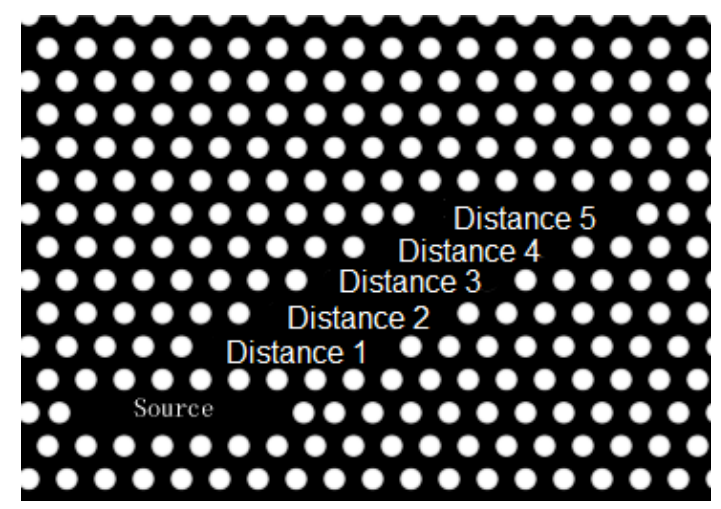

FIGURE 2.10: Relative cavity position varied from distance 1 to distance 5 . 


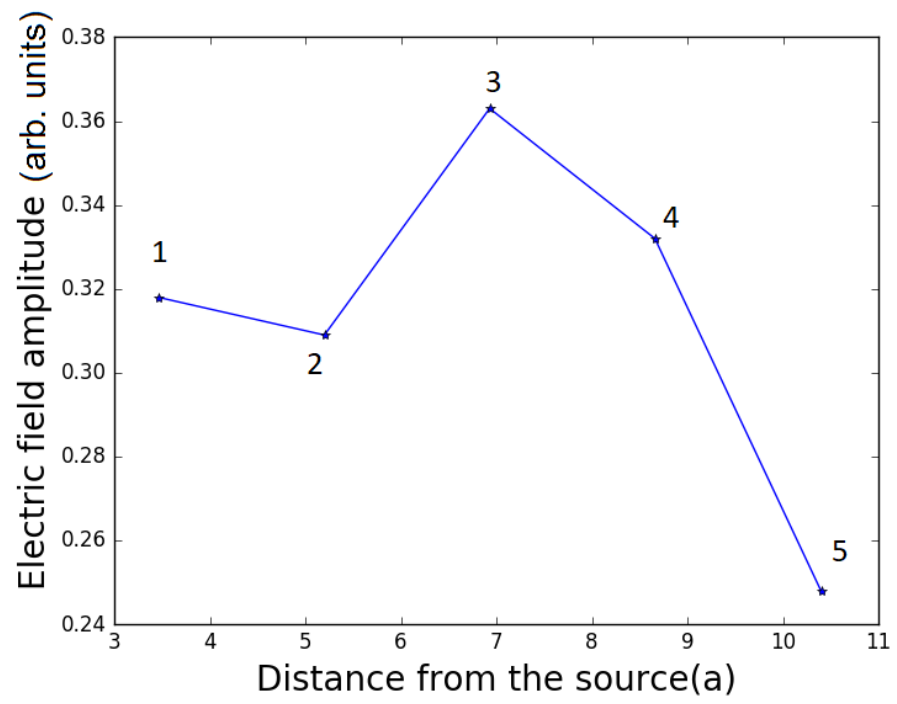

FIGURE 2.11: Plot of electric field vs distance, the unit of distance is a.

From Figure 2.11, we can see there is a peak value around distance 7a. After the peak value, the amplitude of electric field decreases quickly. The peak of this plot is mainly caused by a cavities' location not properly aligned with the leakage direction of photonic cavity.

\subsubsection{ITO based 2D photonic crystal}

Now we apply what we have learned to the experimental photonic crystal made of ITO. The refractive index of ITO is 2.18 [19] (at room temperature), which is lower than the refractive index of silicon. We choose the radius ratio $\mathrm{r} / \mathrm{a}=0.266$. For practical reasons, radius $r=0.266 a$ is easy to fabricate with a focused ion beam (FIB). We use the model cavity of a line defect cavity with five air holes missing. 


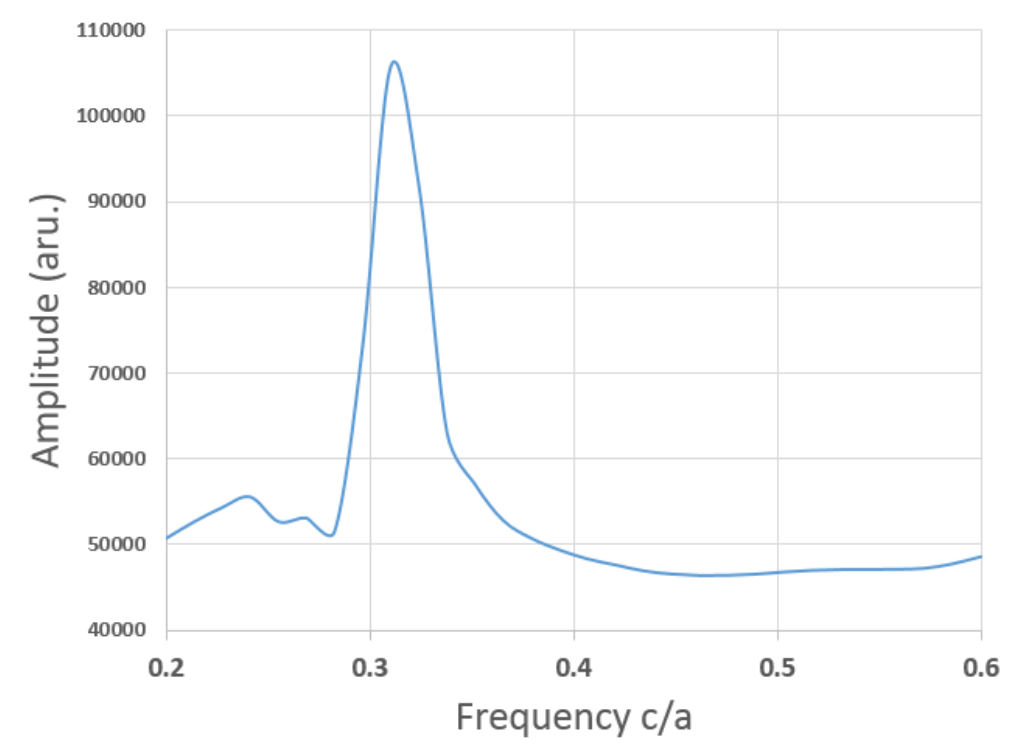

FIGURE 2.12: Spectrum of the L5 photonic cavity with triangular lattice and radius ratio 0.266 . The normalized resonant frequency is determined to be $0.31 \mathrm{c} / \mathrm{a}$ from the peak.

The wavelength in SI units can be determined from

$$
\lambda=\frac{a}{0.31}=\frac{128 \mathrm{~nm}}{0.31}=413 \mathrm{~nm} .
$$

and the frequency is given by

$$
f=\frac{c}{\lambda}=\frac{0.31 c}{a}=0.31 \frac{c}{a} .
$$

If we have $\mathrm{a}=128 \mathrm{~nm}$, then the free space frequency in SI units is

$$
f=\frac{0.31 \times 3 \times 10^{8}}{128 \times 10^{-9}}=7.27 \times 10^{14} s^{-1} .
$$

The simulated result is shown in Figure 2.13. 


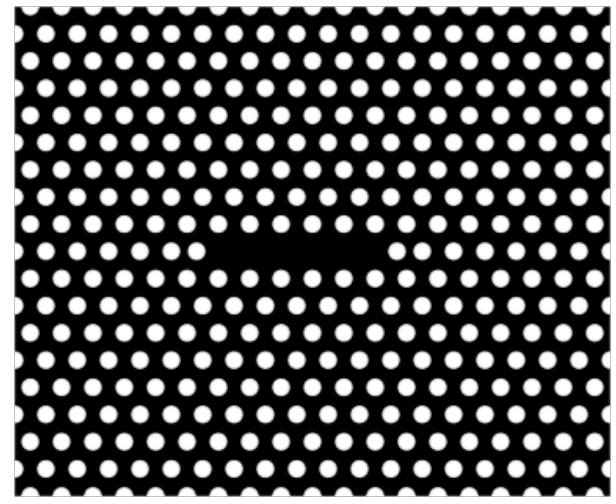

(A)

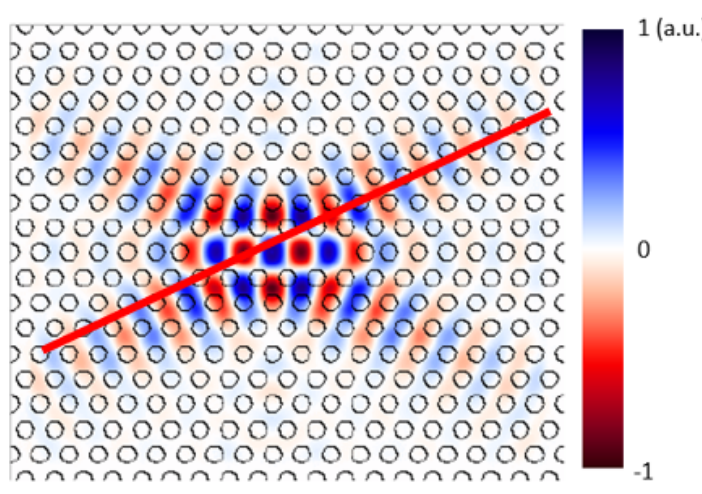

(B)

FIGURE 2.13: (A) Simulation model of L5 photonic cavity; (B) Resonant mode of L5 photonic cavity.

In this simulation the leakage direction indicated by the red line in Figure $2.13 \mathrm{~B}$ is not well-aligned with the cavity positions in the previous study. We therefore expect the highest energy at an angle different than 30 degrees. From Figure 2.13B we can clearly see the distribution of electric fields of a single photonic cavity, the color shows the amplitude of the electric field, blue means negative field strength and red means positive field strength. Energy leaks from each corner of the photonic cavity. To couple two cavities together, we need to have some energy exchange. The second cavity's position is therefore very important. We put the second cavity in the part of the leaking electric field, a line 30 degrees from horizontal direction of the first cavity such that the leaked energy can be transferred to the second cavity. Figure 2.14 is our simulation result for two such cavities. The point source is in center of the lower cavity and the upper cavity doesn't have a source. The boundary condition is absorbing boundary condition. 


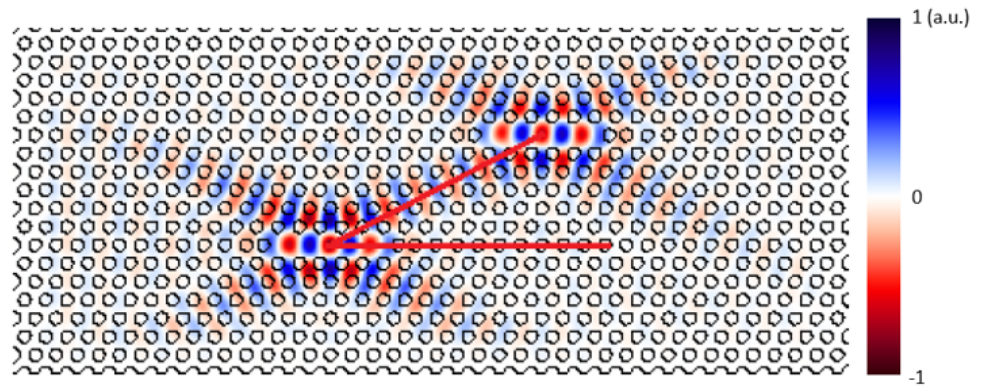

FIGURE 2.14: Two coupled photonic cavity at 30 degrees.

The energy exchange between two photonic cavities can be observed from a time-domain simulation result, as shown in Figure 2.15. The leakage of the photonic cavity is in a direction from a horizontal line that defines the direction of the first oscillator that corresponds to the $\Gamma-K$ direction in the Brillouin zone shown in Figure 1.9. Also in Figure 1.14B, there is no bandgap around the $K$ point, which indicates leakage.
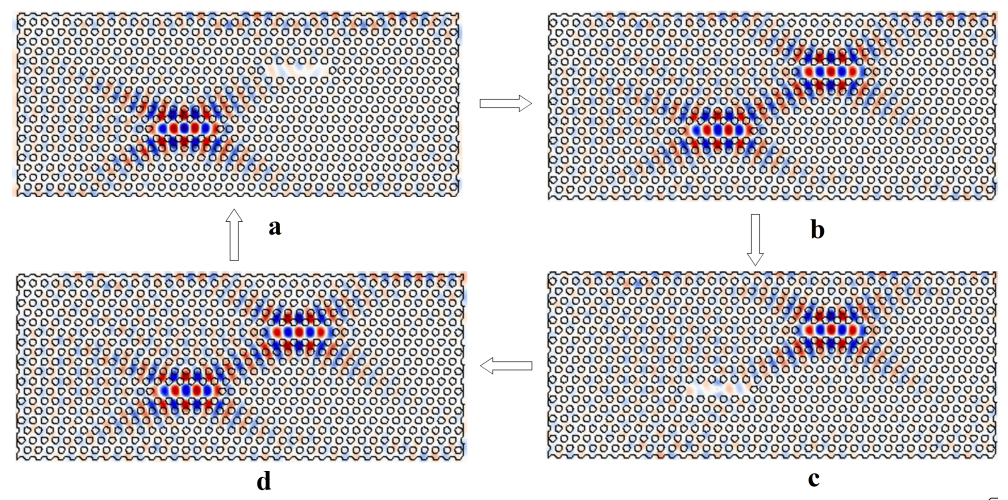

FIGURE 2.15: A time variation of two coupled cavities.

In Figure 2.15, from (a) to (d), each frame is represents a different time in the simulation, the time duration from (a) to (d) and back to (a) is equal one energy transfer period. One period is about $3.3 \mathrm{a} / \mathrm{c}$ in our simulation. The time elapse 
between each frame is $1 / 4$ period. In frame (a), all energy is concentrated in the lower cavity. In Frame (b) partial energy has been transferred to the upper cavity. In Frame (c) all the energy is concentrated in the upper cavity. In Frame (d) partial energy has been transferred to the lower cavity.

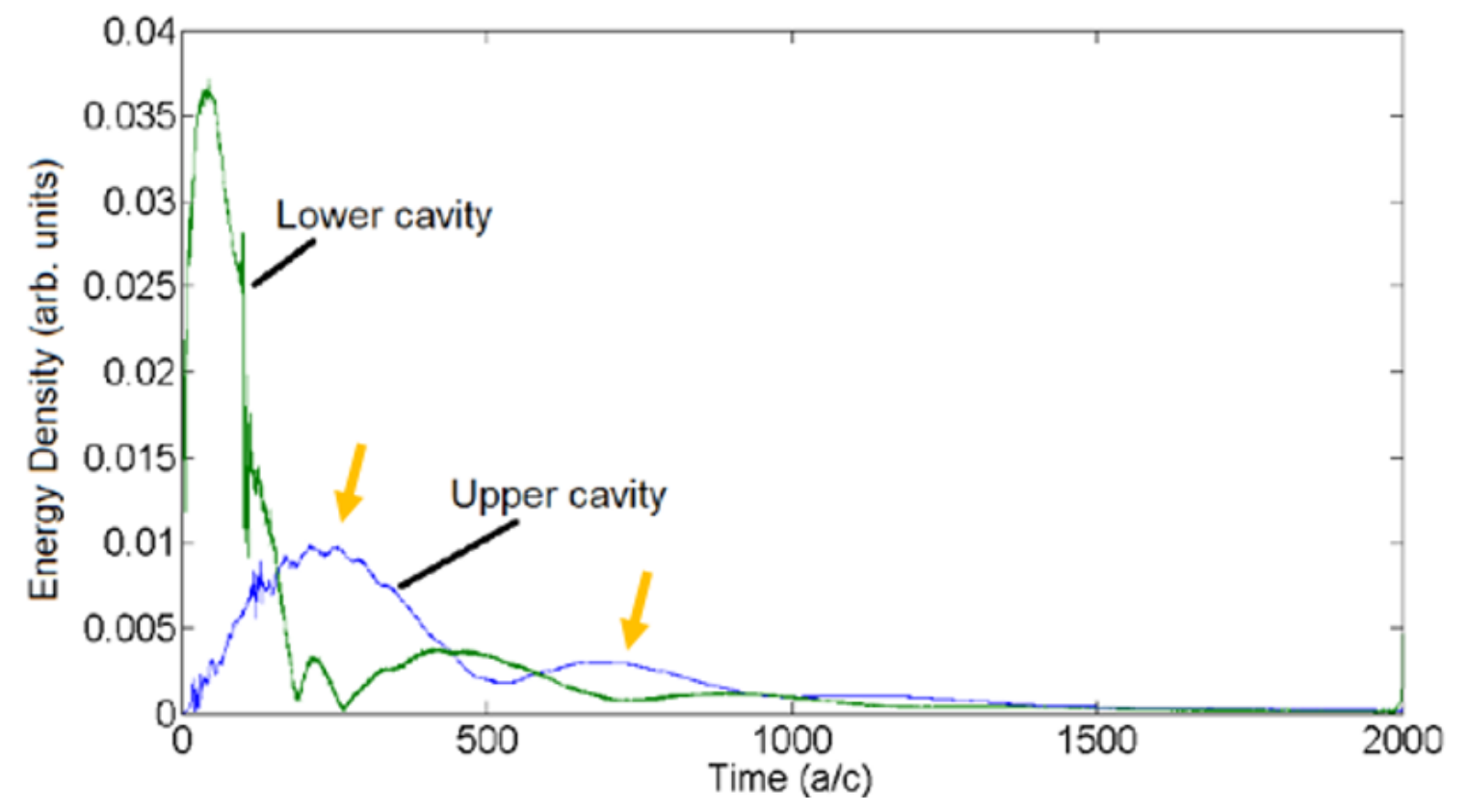

FIGURE 2.16: A plot of upper and lower cavity's energy vs time.

The energy transfer between these two cavities is very similar to the energy transfer between two oscillators. We performed a long FDTD simulation and calculated the average energy inside one cavity over a time of 20c/a. In Figure 2.16 , the blue line is the average energy inside the lower cavity that has a source in it. The green line is the average energy inside the upper cavity that does not have a source in it. The excitation processing occurs from 0 to $150 \mathrm{a} / \mathrm{c}$. Here we use Meep time, where one Meep time unit is equal to a/c. We can convert the Meep 
time unit to real time unit using periodic constant "a" divided by the speed of light. After we removed the source, we then run the simulation until 2000a/c. We can see in Figure 2.16 that the energy of the lower cavity decays very fast and part of the energy is transferred to the upper cavity. The energy of the upper cavity then transfers back to the lower cavity. The phase difference between these two cavities is about 180 degrees. From the plot we can obtain the Rabi frequency. The Rabi frequency is the energy transfer in a two-level system. We can measure the Rabi frequency by measuring the energy transfer period from the Figure 2.16. We can calculate the energy transfer period by measuring the time interval between two peaks in the upper cavity's plot in Figure 2.16. The period we find is about $500 \mathrm{a} / \mathrm{c}$, which is between two yellow arrows in Figure 2.16. The Rabi frequency is

$$
f_{\text {Rabi }}=\frac{1}{T}=\frac{1}{500 a / c}=0.002 c / a .
$$

If we choose $\mathrm{a}=128 \mathrm{~nm}$, the frequency in SI units is,

$$
f_{\text {Rabi }}=\frac{0.002^{8}}{128 \times 10^{-9}}=4.7 \times 10^{12} \mathrm{~s}^{-1} .
$$




\subsubsection{Strong coupling between L5 photonic cavities}

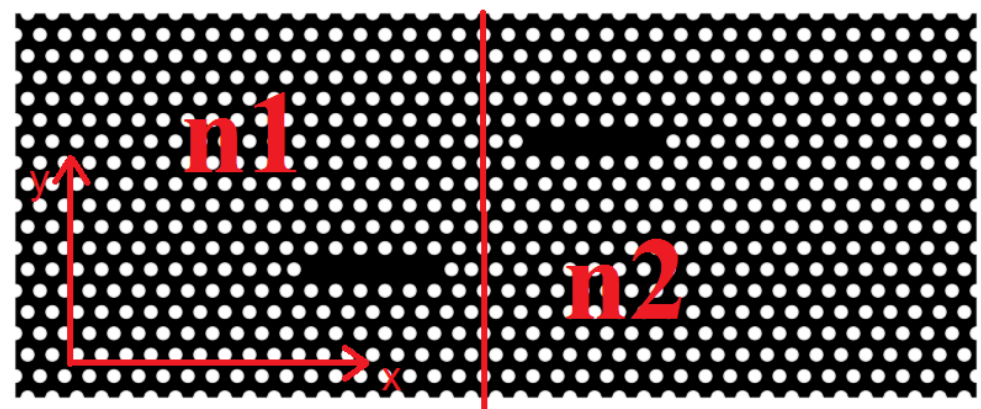

FIGURE 2.17: A simulation model, two cavities at the angle of 30 degrees.

In the previous section we found the angle that produces optimum coupling between the two photonic cavities. To show that the coupling between two photonic crystal cavities is strong coupling, the best way to do so is to produce an anti-crossing plot of strong coupling. Similar to the classical model, instead of tuning the spring constant of one oscillator, we tune the refractive index of one photonic cavity. As indicated in Figure 2.17, the refractive index of the lower cavity is $n_{1}=2.18$, while the refractive index $n_{2}$ of the upper cavity varied between 2.14 and 2.22. We only change the refractive index of the upper cavity. The index of 2.18 is the refractive index of ITO at room temperature. In the simulation, the air hole's radius is $r=0.266 a$. The length of the model is $39 a$, and the height of the model is $15.6 a$. The wavelength is scanned from $2.47 a$ to $2.53 a$. The point electric dipole source is in the center of the lower cavity. The dipole is polarized in the $\mathrm{z}$ direction, the direction normal to the $2 \mathrm{D}$ simulation plane, so it generates a TE mode electromagnetic wave. The vertical distance of these two cavities is six rows, which is equal to 5.2a. The distance in x-direction is nine 
columns from center to center which is equal to $9 a$. Due to the long simulation time duration, the resolution was set to 10 pixel per unit length, which is smaller than $\lambda / 10$.

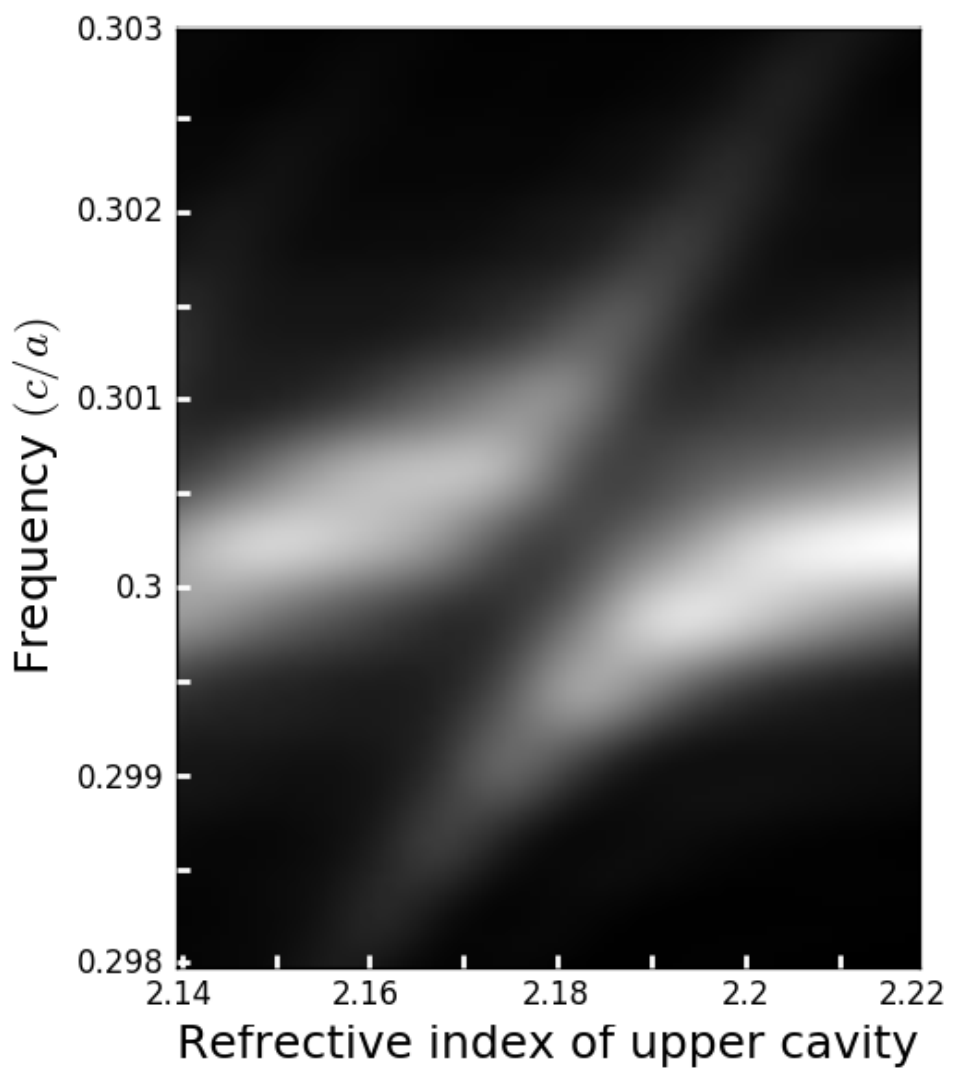

FIGURE 2.18: Anti-crossing image.

By varying the index of one cavity relative to the other we find that we can produce an anti-crossing dispersion relationship between the two cavities that indicates strong coupling (Figure 2.18). The vertical axis is the frequency $a / \lambda$. The horizontal axis is the refractive index of the upper cavity. The brightness in the plot is proportional to the total energy density in the two cavities. The plot shows the anti-crossing behavior in a similar way as in Figure 2.7B. Each vertical line in 
this image represent a frequency scan at the specific refractive index. Figure 2.18 is 512 frequency scans combined together.

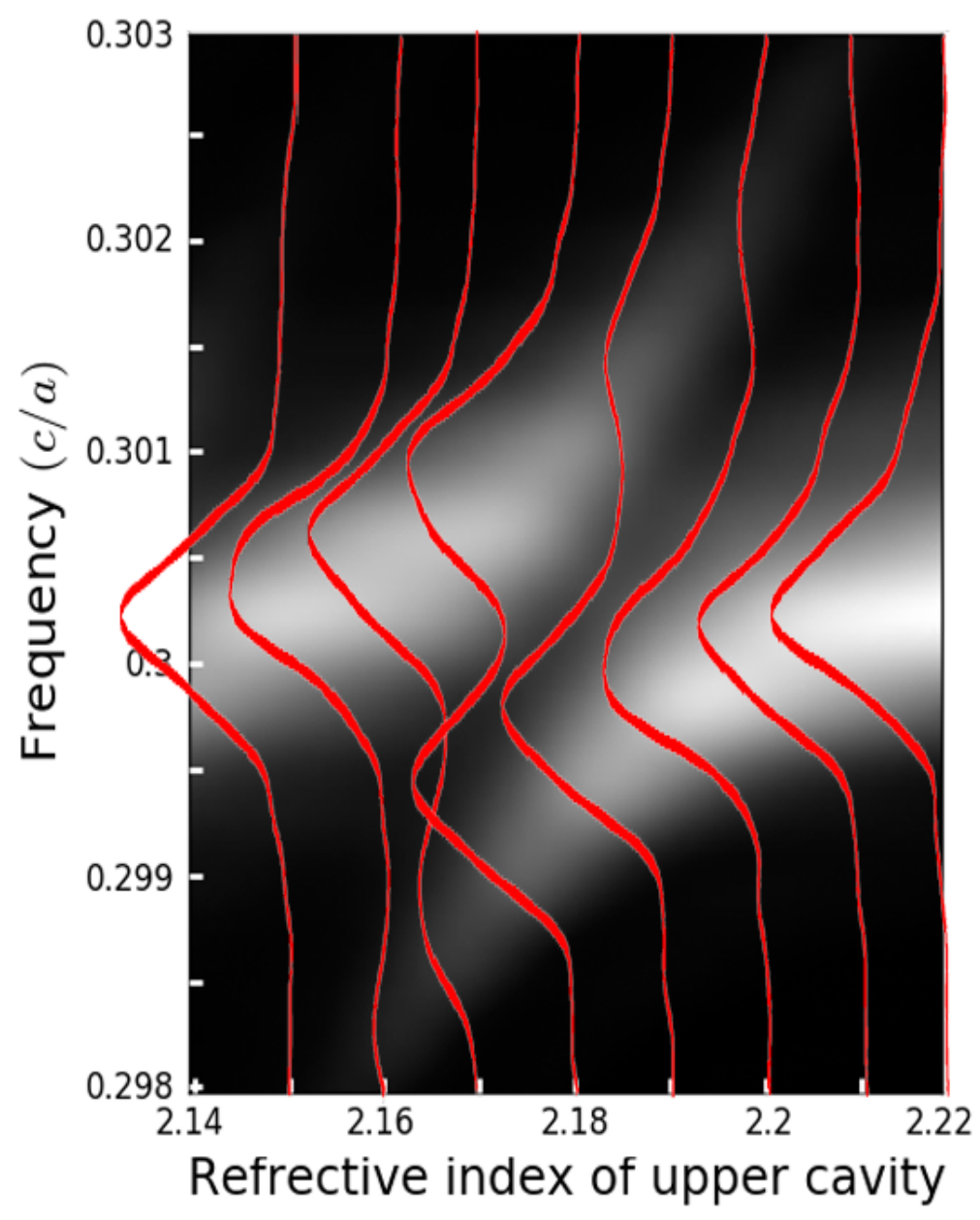

FIGURE 2.19: Anti-crossing image with brightness scale overlap.

Figure 2.19 is the same as Figure 2.18, but here brightness spectra are added for the selected index values of the upper cavity, showing explicitly the spectral behavior of the resonances as two cavities are tuned versus each other. 


\subsubsection{Symmetric and antisymmetric modes}

Another interesting phenomenon is the symmetric and antisymmetric mode of these two cavities. As we already know from Figure 2.19, there are two peaks in the center of the image. The left peak and the right peak have a different modes, which are shown in Figure 2.21. In the left side of the image is a symmetric mode (normalized frequency equal to $2.995 \mathrm{c} / \mathrm{a}$ ) and on the right side of the image is an antisymmetric mode (normalized frequency equal to 3.012c/a) as shown in Figure 2.20. For the symmetric mode, the standing wave inside each cavity has the same phase, but for the antisymmetric mode, the standing wave inside each cavity has a phase difference of 180 degrees.

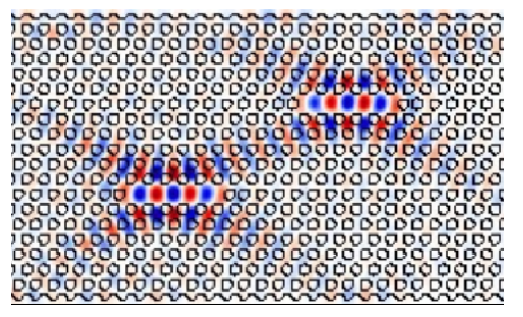

(A)

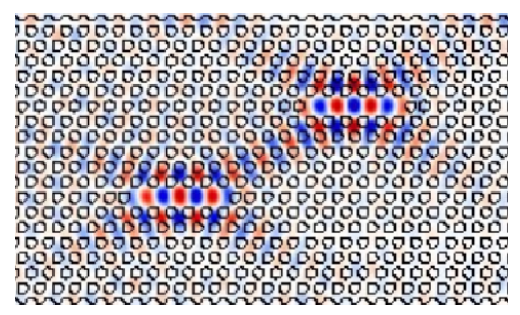

(B)

FIGURE 2.20: Symmetric mode (A); Antisymmetric mode (B). The red color indicates the peak of the electric wave and the blue color indicates the valley of the electric wave.

We can also compare this two modes with a frequency scan when two cavities have same refractive index. In Figure 2.21, there are two peaks. These two peaks represent different modes, which are the symmetric mode and the antisymmetric mode. The left peak is the symmetric mode and the right peak is the antisymmetric mode. 


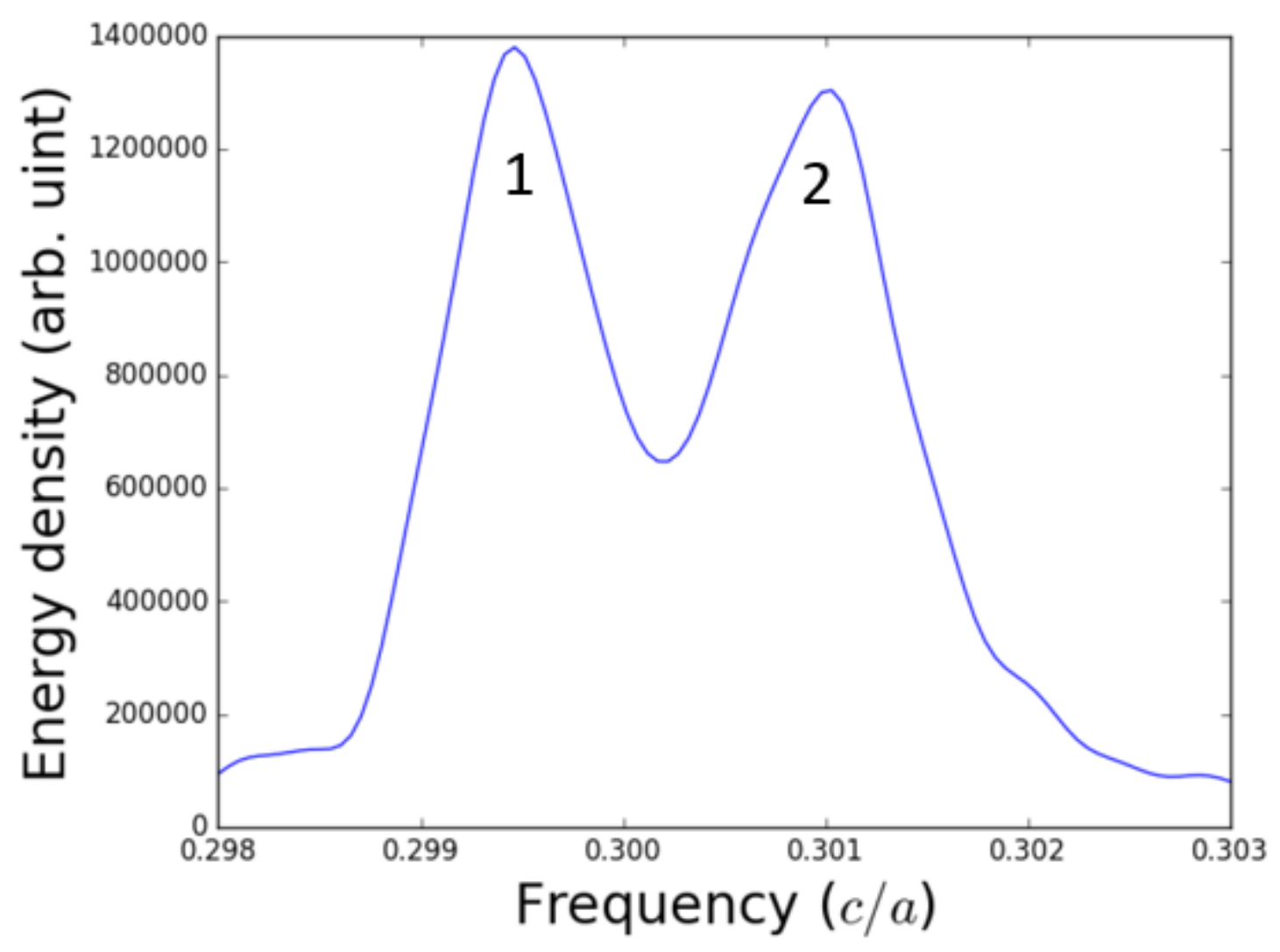

FIGURE 2.21: A frequency scan of the coupling model.

Figure 2.21 shows the combined average energy of the two peak, when the two cavities have same refractive index. We can find the frequency difference between two peaks

$$
\Delta f=0.3012 c / a-0.2995 c / a=0.0017 c / a .
$$

which in SI units is

$$
\Delta f=\frac{0.0017 \times 3 \times 10^{8}}{128 \times 10-9}=4.0 \times 10^{12} \mathrm{~s}^{-1} .
$$

The frequency of these two peaks are $0.2995 \mathrm{c} / \mathrm{a}$ and $0.3012 \mathrm{c} / \mathrm{a}$. These two peaks 
are the two modes of the cavities. Peak 1 is the symmetric mode (Figure 2.20A). Peak 2 is antisymmetric mode (Figure 2.20B). Due to the simulation resolution, the Rabi frequency we get from the Figure 2.21 is not exactly the same as the result we get from Figure 2.16. These two frequencies are different by $15 \%$.

\subsubsection{The fringes in the anti-crossing image}

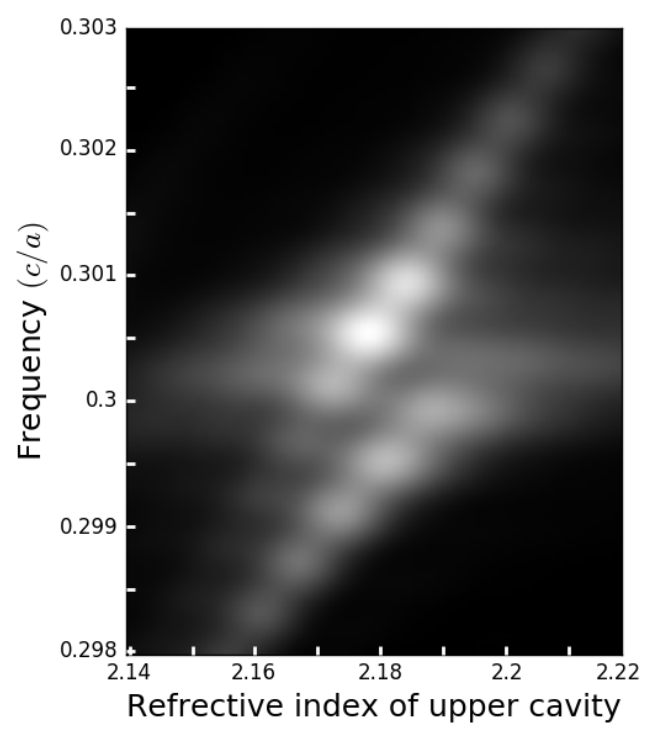

FIGURE 2.22: Fringes in one cavity's anticrossing image.

From the previous section, we noticed that there are some fringes in the single cavity's image as shown in Figure 2.22. The fringes indicate that the distribution of the single cavity's energy vs. the refractive index is not evenly distributed, but in our classical model, the modes distribution of one oscillator and the spring constant should be continuous. Then the fringes may be caused by the reflections from the boundaries. However, all the boundaries of the model have the absorption boundary condition and there should be no reflections from the 
boundaries of the model. We assume the reflection comes from the refractive index boundary between the left part and the right part of the model. To verify our assumptions, we run several additional simulations. First, we remove the upper cavity, so there is no strong coupling effect. Second, we slowly move the boundary from the center of the model to the left edge of the model, as shown in Figure 2.23 and Figure 2.24. At each step, we output an energy distribution of the model. The simulation results show that the fringes in the image become weaker as we move the center boundary away from the center. This confirmed the assumption that the fringes are from the interference between the incident light and reflect light caused by the refractive index boundary.

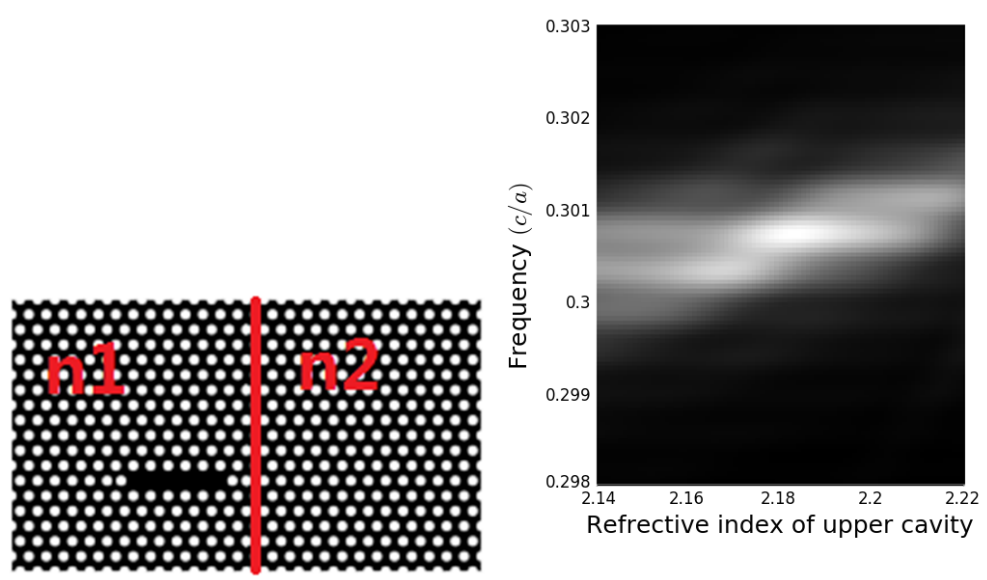

FIGURE 2.23: Remove one cavity. 

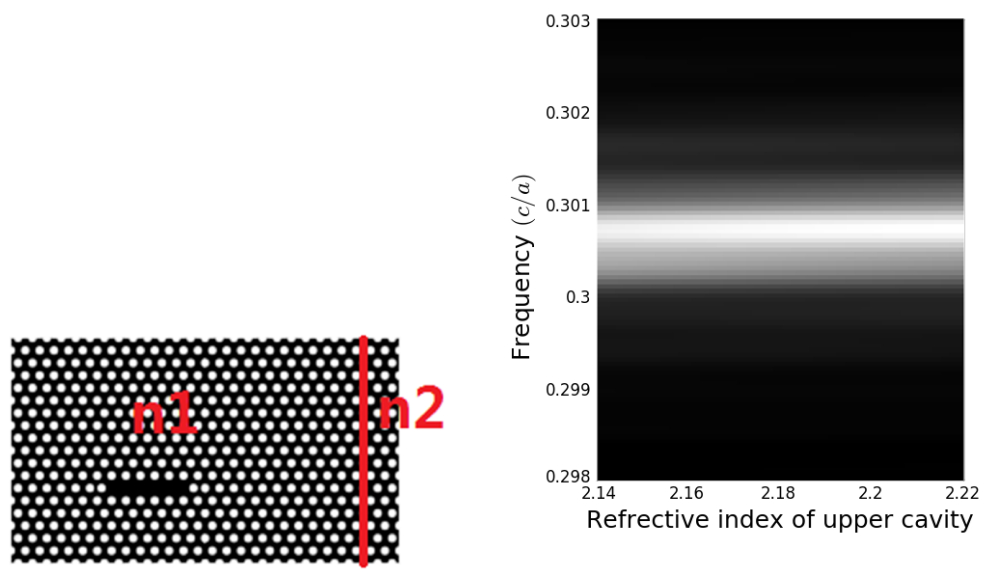

FIGURE 2.24: Move the boundary close to the edge. 


\section{Chapter 3}

\section{Conclusion}

In summary, with the help of a computer-based simulation method, we have studied the properties of the ITO based triangular lattice photonic crystal and the resonant modes of photonic cavities. We verified a TM mode can be strongly coupled. Strong coupling between two photonic crystal cavities has been demonstrated in the finite difference time domain simulation results. To understand the simulation results, we explored the explanation of strong coupling in classical mechanics and compared it to the anti-crossing behavior of the optical cavities. The shape of the anti-crossing image from photonic crystal resembles closely the shape of the classical model. We also observed fringes in the simulation anti-crossing image. The fringes are verified to come from the reflection of the center refractive index boundary.

\subsection{Future work}

This thesis demonstrates a computer-based study of the photonic crystal, but the simulation result still needs to be experimentally verified. In a future experiment we propose to use a PEEM to visualize strong coupling in a photonic crystal. Because of the transparency and conductivity of ITO, it can be used under photoemission electron microscopy (PEEM). PEEM is a type of emission 
microscopy that can visualize the electromagnetic field [20]. With the help of PEEM, we may be able to visualize the strong coupling effect between two photonic crystal cavities at subwavelength scale. We may fabricate the photonic crystal cavities in ITO/glass slabs using a Focused ion beam (FIB). The FIB is capable of Nano prototyping, and it can achieve a 15-nm milling resolution. To experimentally verify our simulation we calculate that for a $400 \mathrm{~nm}$ laser, the radius of air holes should be $34 \mathrm{~nm}$. Holes of this size may not be possible with our present FIB. It may be necessary to locate a higher resolution FIB or explore the near-IR regime. 


\section{Bibliography}

[1] L. Rayleigh, "Xvii. on the maintenance of vibrations by forces of double frequency, and on the propagation of waves through a medium endowed with a periodic structure," The London, Edinburgh, and Dublin Philosophical Magazine and Journal of Science, vol. 24, no. 147, p. 145, 1887.

[2] E. Yablonovitch, "Inhibited spontaneous emission in solid-state physics and electronics," Physical Review Letters, vol. 58, no. 20, p. 2059, 1987.

[3] S. John, "Strong localization of photons in certain disordered dielectric superlattices," Physical Review Letters, vol. 58, no. 23, p. 2486, 1987.

[4] T. F. Krauss, R. M. De La Rue, and S. Brand, "Two-dimensional photonicbandgap structures operating at near-infrared wavelengths," Nature, vol. 383, no. 6602, p. 699, 1996.

[5] P. Russell, "Photonic crystal fibers," Science, vol. 299, no. 5605, p. 358, 2003.

[6] M. Takita, A. D. Córcoles, E. Magesan, B. Abdo, M. Brink, A. Cross, J. M. Chow, and J. M. Gambetta, "Demonstration of weight-four parity measurements in the surface code architecture," Physical Review Letters, vol. 117, no. 21, p. 210505, 2016.

[7] C. Sun, M. T. Wade, Y. Lee, J. S. Orcutt, L. Alloatti, M. S. Georgas, A. S. Waterman, J. M. Shainline, R. R. Avizienis, S. Lin, et al., "Single-chip microprocessor that communicates directly using light," Nature, vol. 528, no. 7583, p. 534, 2015.

[8] K. Yee, "Numerical solution of initial boundary value problems involving maxwell's equations in isotropic media," IEEE Transactions on Antennas and Propagation, vol. 14, no. 3, p. 302, 1966.

[9] A. F. Oskooi, D. Roundy, M. Ibanescu, P. Bermel, J. D. Joannopoulos, and S. G. Johnson, "Meep: A flexible free-software package for electromagnetic simulations by the fdtd method," Computer Physics Communications, vol. 181, no. 3, p. 687, 2010. 
[10] A. Taflove and S. C. Hagness, Computational Electrodynamics. Artech house, 2005.

[11] J. D. Joannopoulos, S. G. Johnson, J. N. Winn, and R. D. Meade, Photonic Crystals: Molding the Flow of Light. Princeton university press, 2011.

[12] E. Matioli and C. Weisbuch, "Impact of photonic crystals on led light extraction efficiency: approaches and limits to vertical structure designs," Journal of Physics D: Applied Physics, vol. 43, no. 35, p. 354005, 2010.

[13] H.-G. Park, S.-H. Kim, S.-H. Kwon, Y.-G. Ju, J.-K. Yang, J.-H. Baek, S.-B. Kim, and Y.-H. Lee, "Electrically driven single-cell photonic crystal laser," Science, vol. 305, no. 5689, pp. 1444-1447, 2004.

[14] P. Yeh, Optical Waves in Layered Media. Wiley, 1988.

[15] R. Bose, D. Sridharan, G. Solomon, and E. Waks, "Observation of strong coupling through transmission modification of a cavity-coupled photonic crystal waveguide," Optics express, vol. 19, no. 6, pp. 5398-5409, 2011.

[16] K. Inoue and K. Ohtaka, Photonic Crystals: Physics, Fabrication and Applications, vol. 94. Springer, 2013.

[17] Y. Akahane, T. Asano, B. S. Song, and S. Noda, "High-q photonic nanocavity in a two-dimensional photonic crystal," Nature, vol. 425, no. 6961, p. 944, 2003.

[18] L. Novotny, "Strong coupling, energy splitting, and level crossings: A classical perspective," American Journal of Physics, vol. 78, no. 11, p. 1199, 2010.

[19] M. N. Polyanskiy, "Refractive index database." https:// refractiveindex. info. Accessed on 2017-11-08.

[20] J. Fitzgerald, R. C. Word, and R. Könenkamp, "Subwavelength visualization of light in thin film waveguides with photoelectrons," Physical Review B, vol. 89, no. 19, p. 195129, 2014. 